\title{
An axisymmetric non-hydrostatic model for double-diffusive water systems
}

\author{
Koen Hilgersom ${ }^{1}$, Marcel Zijlema ${ }^{2}$, and Nick van de Giesen ${ }^{1}$ \\ ${ }^{1}$ Water Resources Section, Faculty of Civil Engineering and Geosciences, Delft University of Technology, Delft, \\ P.O. Box 5048, 2600 GA, the Netherlands \\ ${ }^{2}$ Environmental Fluid Mechanics Section, Faculty of Civil Engineering and Geosciences, Delft University of Technology, \\ Delft, P.O. Box 5048, 2600 GA, the Netherlands
}

Correspondence: Koen Hilgersom (k.p.hilgersom@tudelft.nl)

Received: 6 July 2016 - Discussion started: 13 September 2016

Revised: 26 October 2017 - Accepted: 18 December 2017 - Published: 6 February 2018

\begin{abstract}
The three-dimensional (3-D) modelling of water systems involving double-diffusive processes is challenging due to the large computation times required to solve the flow and transport of constituents. In 3-D systems that approach axisymmetry around a central location, computation times can be reduced by applying a 2-D axisymmetric model set-up. This article applies the Reynolds-averaged NavierStokes equations described in cylindrical coordinates and integrates them to guarantee mass and momentum conservation. The discretized equations are presented in a way that a Cartesian finite-volume model can be easily extended to the developed framework, which is demonstrated by the implementation into a non-hydrostatic free-surface flow model. This model employs temperature- and salinity-dependent densities, molecular diffusivities, and kinematic viscosity. One quantitative case study, based on an analytical solution derived for the radial expansion of a dense water layer, and two qualitative case studies demonstrate a good behaviour of the model for seepage inflows with contrasting salinities and temperatures. Four case studies with respect to doublediffusive processes in a stratified water body demonstrate that turbulent flows are not yet correctly modelled near the interfaces and that an advanced turbulence model is required.
\end{abstract}

\section{Introduction}

Over the past decades, numerical salt and heat transport models have increased their capability to capture patterns of double diffusion on scales varying from laboratory set-ups to the ocean (Yoshida and Nagashima, 2003; Kunze, 2003). Despite the advance in computation power and parallel computing, the requirement of dense grids for the three-dimensional (3-D) modelling of salt and heat transport often yields unacceptable computation times. In this article, we present a framework for a finite-volume approach that allows for freesurface flow modelling in a 2-D axisymmetric grid. The model framework is intended for a shallow water body where salinity and temperature gradients potentially induce doublediffusive processes. As such, the model intends to simulate larger-scale features of double diffusion (i.e., interface locations in a stratified system and heat and salt transport).

Kunze (2003) stresses that numerical and analytical methods to model double diffusion are often only applicable on specific scales. For example in oceans, internal wave shear and strain enhance salt-finger growth, leading to higher salt and heat fluxes over stratified interfaces. Traxler et al. (2011) addresses the issue of scale by describing four modes of instability in salt-fingering systems, which play a role on different scales. Their 3-D simulations of large-scale instability in salt-fingering systems are the first known successful direct numerical simulations (DNSs). Carpenter et al. (2012) were among the first to model systems in the double-diffusive convection regime with 3-D DNS. Their detailed simulations showed that, in this regime, the salt and heat fluxes across the interface are largely governed by molecular diffusion and that these salt and heat diffusion rates control the thickness of the salt and heat interface, respectively. Kimura and Smyth (2007) used 3-D DNS to model salt sheets for a doublediffusively stratified flow interacting with inflectional shear. 
Yoshida and Nagashima (2003) have shown that 2-D numerical models are already well able to simulate small-scale processes in laboratory set-ups. On a larger scale, Sommer et al. (2014) confirm the findings of Carpenter et al. (2012) with 2-D DNS and high-resolution measurements of a double-diffusive staircase in Lake Kivu for density ratios larger than 3, noting that in these systems external turbulence by shear or internal waves should be absent to maintain diffusion as the main driver for salt and heat transport. Noguchi and Niino (2010a, b) used 2-D DNS to study the spontaneous layer formation in the double-diffusive convection regime and explore the layer formation from the nonlinear evolution of disturbances.

Most numerical modelling studies of double-diffusive processes calculate interfaces and salt and heat fluxes on the oceanic scale (Stommel and Fedorov, 1967; Stern, 1967; Ruddick and Gargett, 2003; Kelley et al., 2003; Kunze, 2003; Kimura et al., 2011). This can be explained by the ubiquity of these systems in oceans (Huppert and Turner, 1981) and by the potential of oceanic thermohaline stratification as an energy source (Stommel et al., 1956; Vega, 2002). These larger-scale simulations are commonly performed with Reynolds-averaged Navier-Stokes (RANS) models. For example, Radko et al. (2014a, b) successfully applied a 3D RANS model to an oceanic-scale salt-finger staircase. Recently, the modelling of these phenomena in smallerscale water bodies has started to be developed. For example, double-diffusive processes like thermohaline staircasing have been successfully modelled in lakes (Schmid et al., 2003), although these systems are generally modelled with analytical or empirical formulations (Kelley et al., 2003; Schmid et al., 2004; Arnon et al., 2014). Other known numerical modelling studies consider double-diffusive convection in monitoring wells (Berthold and Börner, 2008) and the collection of thermal energy in solar ponds (Cathcart and Wheaton, 1987; Giestas et al., 2009; Suárez et al., 2010, 2014). However, modelling these complex physical processes in shallow waters still imposes a major scientific and computational challenge (Dias and Lopes, 2006).

Axisymmetric CFD models are applied in a wide variety of fields. Examples of applications include the modelling of gas flow past a gravitating body in astronomy (Shima et al., 1985), radiative heat transfer in cylindrical enclosures (Menguc and Viskanta, 1986), the heating of air flowing through a combustion burner (Galletti et al., 2007), and acoustic axisymmetric waves in elastic media (Schubert et al., 1998). The similarity between these examples is that a model calculating in two spatial dimensions models 3-D processes due to axisymmetry. In geohydrology, axisymmetric models are often applied for groundwater flow around injection and abstraction wells (Bennett et al., 1990). Groundwater modelling software often offers code extensions that adjust several input parameters to allow for such modelling approaches (Reilly and Harbaugh, 1993; Langevin, 2008).
In some cases, axisymmetric grid set-ups can also be preferential for hydrodynamic surface water models. Examples of such cases are close-to-circular water bodies with uniform boundaries and the flow around a central point (e.g., a local inflow from a pipe or groundwater seepage). The occurrence of local saline seepage inflows into shallow water bodies of contrasting temperatures has been described by De Louw et al. (2013). Hilgersom et al. (2016) have shown how these local inflows can induce thermohaline stratification in the shallow surface water bodies above these inflows.

This article derives a framework for an axisymmetric freesurface RANS model, which is implemented in SWASH. SWASH is an open-source non-hydrostatic modelling code for the simulation of coastal flows including baroclinic forcing (SWASH source code, 2011). It is suitable for the simulation of flows and transport in varying density fields because (1) the staggered grid allows for a momentum and mass conservative solution of the governing equations, which is required for accurate salt and heat transport modelling, and (2) the non-hydrostatic pressure terms aid the simulation of flows in fields with large density variations. Another major advantage of SWASH is the flexible and easily extendible code, which can be applied for free under the GNU GPL licence. Other properties of SWASH are the opportunity to apply terrain-following $\sigma$ layers for the definition of cell depths and the user friendly pre- and post-processing.

The development of an axisymmetric variation of SWASH falls in line with our research on localized saline water seepage in Dutch polders. To simulate the effect of a local seepage inflow on the temperature profile of the surface water body, a numerical model is required that accounts for sharp density gradients, a free surface, and potential double-diffusive processes. The axisymmetric grid set-up aids in correctly representing the volumetric inflow and modelling the flow processes around the local inflow. Rather than a fine-scale model that resolves all the relevant scales for double-diffusive processes, the current approach should be regarded as a pragmatic solution tool that can be run on relatively simple computer infrastructure.

In this article, we present the resulting numerical framework to extend a 2-D finite-volume model into a 2-D axisymmetric model by adding a few terms to the solution of the governing Navier-Stokes and transport equations. These terms are implemented in the SWASH code. The model code is further extended with a new transport module calculating salt and heat transfer. Although the model generally calculates with a mesh size that is larger than the size required to solve small-scale double-diffusive instabilities, the aim is to allow the model to approximate interface locations and salt and heat fluxes. The functioning of the code is validated with case studies involving different salinity and temperature gradients. 


\section{Method}

\subsection{Governing equations}

The governing equations in this study are the Reynoldsaveraged Navier-Stokes equations for the flow of an incompressible fluid derived in cylindrical coordinates $(r, \alpha, z)$ (Batchelor, 1967). Due to point symmetry, the gradients in the tangential direction $(\alpha)$ are set to zero, which leaves the solution of the equations in one horizontal and one vertical dimension (i.e., 2-DV).

$$
\begin{aligned}
& \frac{1}{r} \frac{\partial u r}{\partial r}+\frac{\partial w}{\partial z}=0 \\
& \frac{\partial u}{\partial t}+\frac{\partial u u}{\partial r}+\frac{\partial w u}{\partial z}= \\
& -\frac{1}{\rho} \frac{\partial p}{\partial r}+\left(\frac{1}{r} \frac{\partial}{\partial r}\left(v_{\mathrm{h}} r \frac{\partial u}{\partial r}\right)-\frac{v_{\mathrm{h}} u}{r^{2}}+\frac{\partial}{\partial z}\left(v_{\mathrm{v}} \frac{\partial u}{\partial z}\right)\right) \\
& \frac{\partial w}{\partial t}+\frac{\partial u w}{\partial r}+\frac{\partial w w}{\partial z}= \\
& -\frac{1}{\rho} \frac{\partial p}{\partial z}+\frac{1}{r} \frac{\partial}{\partial r}\left(v_{\mathrm{h}} r \frac{\partial w}{\partial r}\right)+\frac{\partial}{\partial z}\left(v_{\mathrm{v}} \frac{\partial w}{\partial z}\right)-g
\end{aligned}
$$

In these equations, $r$ represents the horizontal axis in the radial direction and $z$ the vertical axis, with $u$ and $w$ the velocities along these axes, respectively. The density $\rho$ is calculated from the local temperature and salinity states by the updated Eckart formula (Eckart, 1958; Wright, 1997), which is based on the UNESCO IES80 formula (UNESCO, 1981).

This RANS model allows for turbulence modelling with the standard $k-\epsilon$ model (Launder and Spalding, 1974). This article presents cases that are modelled with and without this turbulence model. In the case of the former, the modelled eddy viscosity is added to the molecular viscosity, yielding a non-uniform vertical viscosity $v_{\mathrm{v}}$. For all the calculations, the horizontal kinematic viscosity $v_{\mathrm{h}}$ is set uniform to its molecular value $\left(\sim 10^{-6} \mathrm{~m}^{2} \mathrm{~s}^{-1}\right)$.

The pressure terms are split into hydrostatic and hydrodynamic terms according to Casulli and Stelling (1998):

$$
\begin{aligned}
& \frac{1}{\rho} \frac{\partial p}{\partial r}=\frac{g}{\rho} \frac{\partial \int_{z^{\prime}=z}^{\zeta} \rho\left(r, z^{\prime}, t\right) d z^{\prime}}{\partial r}+\frac{\partial q}{\partial r}, \\
& \frac{1}{\rho} \frac{\partial p}{\partial z}+g \equiv \frac{\partial q}{\partial z},
\end{aligned}
$$

where $q$ denotes the hydrodynamic pressure component and $\zeta$ the local free-surface level relative to the reference plane (Fig. 1). Horizontal variations in atmospheric pressure are neglected. The first right-hand-side term of Eq. (4) is split into baroclinic and barotropic components when the equations are integrated over the cell depth in Sect. 2.3. In the vertical, the baroclinic pressure gradient and the gravitational acceleration cancel each other out, leaving the hydrodynamic pressure gradient (Eq. 5).

The free surface is calculated according to Zijlema and Stelling (2008) by integrating Eq. (1) over the depth of

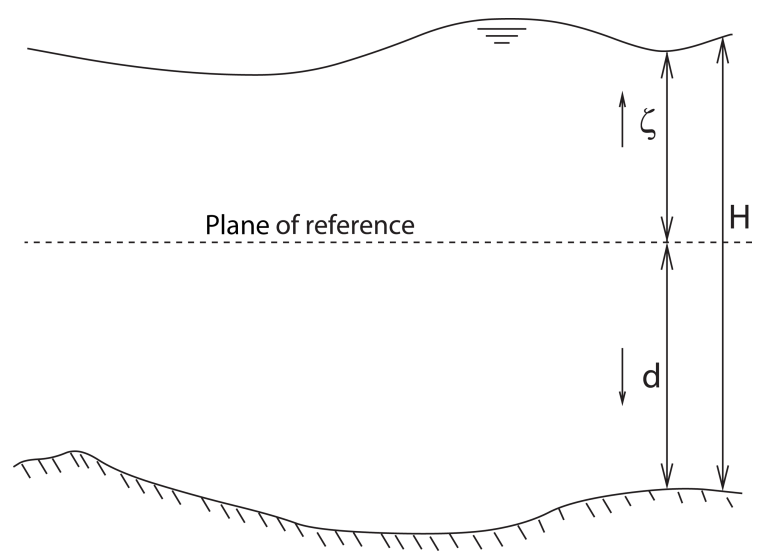

Figure 1. Definition of the free-surface level $\zeta$ and the bottom level $d$ (Zijlema and Stelling, 2005).

the water column and applying the free-surface condition $\left.w\right|_{z=\zeta}=\partial \zeta / \partial t+u \partial \zeta / \partial r$ :

$\frac{\partial \zeta}{\partial t}+\frac{1}{r} \frac{\partial r \bar{Q}}{\partial r}=0 \quad \bar{Q} \equiv U H=\int_{-d}^{\zeta} u d z$,

where $U$ is the depth-averaged velocity, and $d$ is the local bottom depth (Fig. 1). $\bar{Q}$ represents the radial discharge per unit tangential width.

The transport of mass and heat is calculated with the convection-diffusion equation:

$\frac{\partial c}{\partial t}+\frac{1}{r} \frac{\partial r u c}{\partial r}+\frac{\partial w c}{\partial z}=\frac{1}{r} \frac{\partial}{\partial r}\left(D_{\mathrm{h}} r \frac{\partial c}{\partial r}\right)+\frac{\partial}{\partial z}\left(D_{\mathrm{v}} \frac{\partial c}{\partial r}\right)$,

where the concentration $c$ represents either the salinity $S$ or temperature $T$.

In the case that turbulence is modelled, the vertical turbulent diffusion, $D_{\mathrm{v}}$, is calculated by adding the molecular diffusivity and turbulent diffusivity: $D_{\mathrm{v}}=D_{\mathrm{mol}}+D_{\text {turb }}$. The turbulent diffusivity is calculated by dividing the eddy viscosity $v_{\text {turb }}$ by the turbulent Prandtl number $(P r=0.85)$ in the case of heat transport or by the turbulent Schmidt number $(S c=0.7)$ in the case of salt transport:

$D_{\text {turb; }}=\frac{\nu_{\text {turb }}}{P r}=\frac{\nu_{\text {turb }}}{0.85}$,

$D_{\text {turb; } \mathrm{S}}=\frac{v_{\text {turb }}}{S c}=\frac{v_{\text {turb }}}{0.7}$,

with $D_{\text {turb; T }}$ and $D_{\text {turb; }}$ being the thermal and solutal turbulent diffusivities in $\mathrm{m}^{2} \mathrm{~s}^{-1}$, respectively.

In non-turbulent thermohaline systems, stability largely depends on density gradients and molecular heat and salt diffusion rates, which in turn are highly dependent on temperature and salinity. The heat and salt diffusivities are related to temperature $T\left({ }^{\circ} \mathrm{C}\right)$ and salinity $S$ (weight $-\%$ ) by a quadratic regression on data presented in the International Critical Tables of Numerical Data, Physics, Chemistry and Technology 


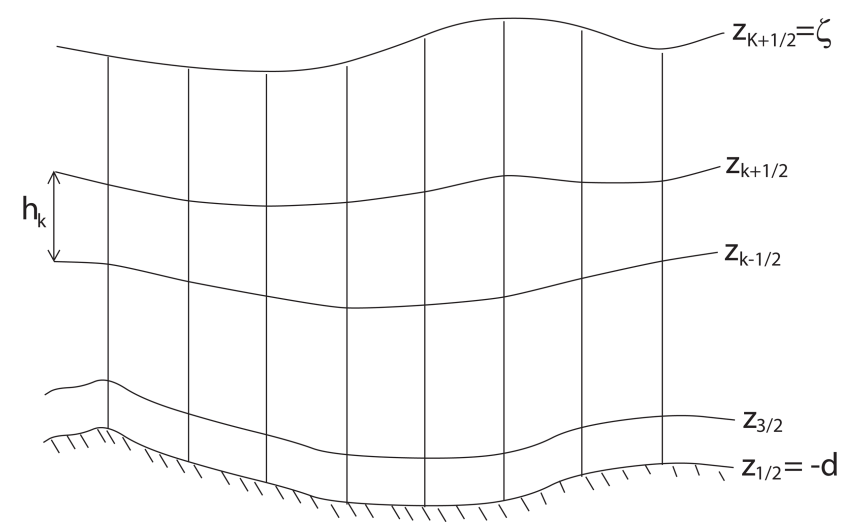

Figure 2. Vertical grid definition (sigma layers) (Zijlema and Stelling, 2005).

(Washburn and West, 1933).

$$
\begin{aligned}
& D_{\text {mol } ;}=1.31721+4.26657 \times 10^{-3} \times T-1.09237 \\
& \times 10^{-6} \times T^{2}+1.74051 \times 10^{-2} \times S-3.17759 \times 10^{-4} \times S^{2} \\
& D_{\text {mol } ;} S=7.66025+2.33023 \times 10^{-1} \times T+3.21974 \\
& \times 10^{-3} \times T^{2}-2.18290 \times 10^{-1} \times S+1.34431 \times 10^{-2} \times S^{2}
\end{aligned}
$$

\subsection{Boundary conditions}

At the free surface, we assume no wind and $\left.q\right|_{z=\zeta}=0$. At the bottom boundary, the vertical velocity is calculated by imposing the kinematic condition $\left.w\right|_{z=-d}=-u \partial d / \partial r$. The presented case studies (Sect. 3) include a local seepage inflow at the bottom boundary, for which the seepage velocity is added to the kinematic condition. For horizontal momentum, the bottom friction is imposed by applying a constant friction coefficient to the bottom layer or by using the logarithmic wall law in case the standard $k-\epsilon$ model is employed, applying a Nikuradse roughness height to determine the amount of friction (Launder and Spalding, 1974).

A special case is the inner boundary at which symmetry occurs; for all variables, the gradient is set to zero, except for horizontal momentum: $\left.u\right|_{r=0}=0$. For the presented case studies, we define a Dirichlet boundary condition for $u$ momentum at the outer boundary at which the total outflow is equated to the instantaneous seepage inflow.

For the transport equation, a homogeneous Neumann boundary condition is defined at each boundary $\left(\frac{\partial c r}{\partial r}=0\right.$ and $\frac{\partial c}{\partial z}=0$ ), except at a defined seepage inflow of known temperature and salt concentration for which a Dirichlet boundary condition is imposed.

\subsection{Numerical framework and implementation}

The physical domain is discretized with a fixed cell width in the radial direction. The width of the cells in the tangential direction increases by a fixed angle $\alpha$, which allows us to consider the horizontal grid as a pie slice (Fig. 3). In the

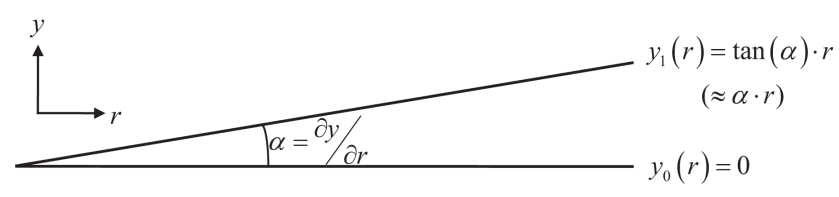

Figure 3. Axis definition.

model, $\alpha$ could be assigned any value (i.e., also $2 \pi$ for a completely circular grid). However, to allow for a simple presentation of the integration step in this subsection, we consider $\alpha$ as a small angle.

For the vertical grid, sigma layering is employed, although part of the layers can be defined by a fixed cell depth (Fig. 2). A classical staggered grid is applied with velocities defined at the cell boundaries and the other states in the cell centre.

For reasons of momentum and mass conservation, Zijlema and Stelling (2005) integrated the governing equations over the cell depth using the Leibniz integral rule (Appendix A). In our case, the cell width in the tangential direction varies as well. Therefore, the equations are integrated over the cell depth and the width in the tangential direction, which is in this case defined as the $y$ dimension (cf. Figs. 2 and 3):

$$
\begin{aligned}
& \int_{z_{k-\frac{1}{2}}}^{z_{k+\frac{1}{2}}} \int_{y_{0}}^{y_{1}}\left(\frac{1}{r} \frac{\partial u r}{\partial r}+\frac{\partial w}{\partial z}\right) \mathrm{d} y \mathrm{~d} z= \\
& \frac{\partial \phi_{k} y_{1}}{\partial r}+y_{1} \omega_{k+\frac{1}{2}}-y_{1} \omega_{k-\frac{1}{2}}+y_{1} \frac{\partial h_{k}}{\partial t}=0,
\end{aligned}
$$

with $\phi_{k}=u_{k} \times h_{k}$ as the cell-depth-integrated velocity and the relative vertical velocity $\omega$ as defined in Eq. (16) of Zijlema and Stelling (2005).

The momentum equations and the transport equation are integrated in a similar fashion.

$$
\begin{aligned}
& {\overline{y_{1}}}^{r} \frac{\partial u_{k}{\overline{h_{k}}}^{r}}{\partial t}+\frac{\partial{\overline{\phi_{k}}}^{r} \hat{u}_{k}{\overline{y_{1}}}^{r}}{\partial r}+{\overrightarrow{y_{1}}}_{\hat{u}_{k+\frac{1}{2}}}{\overline{\omega_{k+\frac{1}{2}}}}^{r} \\
& -\vec{y}_{1} \hat{u}_{k-\frac{1}{2}}{\overline{\omega_{k-\frac{1}{2}}}}^{r}-\alpha \phi_{k} u_{k}+g{\overline{h_{k}}}^{r}{\overline{y_{1}}}^{r} \frac{\partial \zeta}{\partial r}+\frac{\partial q_{k} h_{k} y_{1}}{\partial r} \\
& -\bar{y}_{1} r \bar{q} r z \frac{\partial z_{i+\frac{1}{2}, k+\frac{1}{2}}}{\partial r}+\bar{y}_{1} r \bar{q}^{r z} \frac{\partial z_{i+\frac{1}{2}, k-\frac{1}{2}}}{\partial r} \\
& -\alpha{\overline{h_{k}}}^{r}{\overline{q_{k}}}^{r}+{\overline{y_{1}}}^{r} \frac{g}{\overline{\rho_{k}}} \frac{\partial \overline{\rho_{k}}}{\partial r} \frac{\left(\overline{h_{k}}\right)^{2}}{2}+\frac{g{\overline{h_{k}}}^{r} \overline{y_{1}}}{\overline{\rho_{k}}} \sum_{j=1}^{k-1} \\
& \left(\bar{h}_{j}^{r} \frac{\partial \overline{\rho_{j}}}{\partial r}+\left(\overline{\rho_{j}}-\overline{\rho_{k}}\right) \frac{\partial h_{j}}{\partial r}\right)-\frac{\partial}{\partial r} v_{\mathrm{h}} h_{k} y_{1} \frac{\partial \overline{u_{k}}}{\partial r} \\
& +\alpha h_{k} u_{k} \frac{\nu_{\mathrm{h}}}{r}-{\overline{y_{1}}}^{r}\left[\nu_{\mathrm{v}} \frac{\partial u}{\partial z}\right]_{k-\frac{1}{2}}^{k+\frac{1}{2}}=0
\end{aligned}
$$




$$
\begin{aligned}
& y_{1} \frac{\partial w_{k+\frac{1}{2}}{\overline{h_{k+\frac{1}{2}}}}^{z}}{\partial t}+\frac{\partial \hat{w}_{k+\frac{1}{2}}{\overline{\phi_{k+\frac{1}{2}}}}^{z} y_{1}}{\partial r}-\alpha{\overline{\bar{\phi}_{k+\frac{1}{2}}}}^{r z} w_{k+\frac{1}{2}} \\
& +y_{1} \hat{w}_{k+1}{\overline{\omega_{k+1}}}^{z}-y_{1} \hat{w}_{k}{\overline{\omega_{k}}}^{z}+y_{1} q_{k+1}-y_{1} q_{k} \\
& -\frac{\partial}{\partial r} v_{\mathrm{h}} \bar{y}_{1} r \frac{h_{k+\frac{1}{2}}}{r z} \frac{\partial \bar{w}}{\partial r}-y_{1}\left[v_{\mathrm{v}} \frac{\partial w}{\partial z}\right]_{k}^{k+1}=0 \\
& y_{1} \frac{\partial c_{k} h_{k}}{\partial t}+\frac{\partial \phi_{k} c_{k} y_{1}}{\partial r}+y_{1} \omega_{k+\frac{1}{2}} \hat{c}_{k+\frac{1}{2}}-y_{1} \omega_{k-\frac{1}{2}} \hat{c}_{k-\frac{1}{2}} \\
& -\frac{\partial}{\partial r^{\prime}}\left\{D_{\mathrm{h}}{\overline{y_{1}}}^{r} \bar{h}_{k}^{r} \frac{\partial c}{\partial r^{\prime}}\right\}-y_{1}\left[D_{\mathrm{v}} \frac{\partial c}{\partial z^{\prime}}\right]_{z_{k-\frac{1}{2}}}^{z_{k+\frac{1}{2}}} \\
& +\frac{\partial}{\partial r^{\prime}}\left\{D_{\mathrm{h}} \bar{y}_{1} r \bar{h}_{k}^{r} \frac{\partial \bar{c}_{k}^{r}}{\partial z^{\prime}} \frac{\partial \bar{z}^{z}}{\partial r^{\prime}}\right\}+y_{1}\left[D_{\mathrm{h}} \frac{\partial z_{k}}{\partial r} \frac{\partial c}{\partial r^{\prime}}\right]_{z_{k-\frac{1}{2}}}^{z_{k+\frac{1}{2}}} \\
& -y_{1}\left[D_{\mathrm{h}}\left(\frac{\partial z}{\partial r}\right)^{2} \frac{\partial c}{\partial z^{\prime}}\right]_{z_{k-\frac{1}{2}}}^{z_{k+\frac{1}{2}}}=0
\end{aligned}
$$

Overlined variables denote spatially averaged values for these variables in $r$ or $z$ directions, and arrows denote the use of values from upstream cells. The boxes mark the alpha terms, which are the additional angular terms compared to the 2-DV solutions for the momentum equations in Cartesian coordinates. In the integrated transport equation (Eq. 15), the latter three terms on the left-hand side are the so-called anticreepage terms, which should be incorporated for the calculation of transport when large gradients in water depth occur.

Since $u$ and $w$ are the primitive variables in the momentum equations, and not $u h$ and $w h$ as in Eqs. (13) and (14), we further rewrite the momentum equations according to $\mathrm{Zi}$ jlema and Stelling (2008). In order to do this for the $u$ momentum equation, we first spatially discretize the continuity equation in point $i+\frac{1}{2}$.

$$
\begin{aligned}
& \bar{y}_{1 ; i+\frac{1}{2}} r \frac{\partial \bar{h}_{i+\frac{1}{2}, k}^{r}}{\partial t}+\frac{y_{1 ; i+1} \bar{\phi}_{i+1, k}^{r}-y_{1 ; i} \bar{\phi}_{i, k}^{r}}{\Delta r} \\
& +\bar{y}_{1 ; i+\frac{1}{2}}^{r}\left(\bar{\omega}_{i+\frac{1}{2}, k+\frac{1}{2}}^{r}-\bar{\omega}_{i+\frac{1}{2}, k-\frac{1}{2}}^{r}\right)=0
\end{aligned}
$$

We then spatially discretize the $u$ momentum equation and expand $\partial u_{k} h_{k} / \partial t$ to $h_{k} \partial u_{k} / \partial t+u_{k} \partial h_{k} / \partial t$. The latter term falls out by subtracting Eq. (16) multiplied by $u_{k}$ from Eq. (13).

$$
\begin{aligned}
& \bar{y}_{1 ; i+\frac{1}{2}}^{r} \bar{h}_{i+\frac{1}{2}, k}^{r} \frac{\partial u_{i+\frac{1}{2}, k}}{\partial t} \\
& +\frac{\bar{\phi}_{i+1, k}^{r} \bar{y}_{1 ; i+1}^{r}\left(\hat{u}_{i+1, k}-u_{i+\frac{1}{2}, k}\right)-\bar{\phi}_{i+1, k}^{r} \bar{y}_{1 ; i+1}^{r}\left(\hat{u}_{i+1, k}-u_{i+\frac{1}{2}, k}\right)}{\Delta r} \\
& +\vec{y}_{1 ; i+\frac{1}{2}} \hat{u}_{i+\frac{1}{2}, k+\frac{1}{2}} \bar{\omega}_{i+\frac{1}{2}, k+\frac{1}{2}}^{r}-\vec{y}_{1 ; i+\frac{1}{2}} \hat{u}_{i+\frac{1}{2}, k-\frac{1}{2}} \bar{\omega}_{i+\frac{1}{2}, k-\frac{1}{2}}^{r} \\
& -\alpha \phi_{i+\frac{1}{2}, k} u_{i+\frac{1}{2}, k}+g \bar{h}_{i+\frac{1}{2}, k}^{r} \bar{y}_{1 ; i+\frac{1}{2}}^{r} \frac{\zeta_{i+1}-\zeta_{i}}{\Delta r} \\
& +\frac{q_{i+1, k} h_{i+1, k} y_{1 ; i+1}-q_{i, k} h_{i, k} y_{1 ; i}}{\Delta r}-\bar{y}_{1 ; i+\frac{1}{2}}^{r} \bar{q}_{i+\frac{1}{2}, k+\frac{1}{2}}^{r z} \\
& \frac{z_{i+1, k+\frac{1}{2}}-z_{i, k+\frac{1}{2}}}{\Delta r}+\bar{y}_{1 ; i+\frac{1}{2}}^{r} \bar{q}_{i+\frac{1}{2}, k-\frac{1}{2}}^{r z} \frac{z_{i+1, k-\frac{1}{2}}-z_{i, k-\frac{1}{2}}}{\Delta r} \\
& -\alpha \phi_{i+\frac{1}{2}, k} u_{i+\frac{1}{2}, k}+g \bar{h}_{i+\frac{1}{2}, k}^{r} \bar{y}_{1 ; i+\frac{1}{2}}^{r} \frac{\zeta_{i+1}-\zeta_{i}}{\Delta r} \\
& +\frac{g \bar{h}_{i+\frac{1}{2}, k}^{r} \bar{y}_{1 ; i+\frac{1}{2}}^{r}}{\bar{\rho}_{i+\frac{1}{2}, k}^{r}} \sum_{j=1}^{k-1}\left(\bar{h}_{i+\frac{1}{2}, j}^{r} \frac{\rho_{i+1, j}-\rho_{i, j}}{\Delta r}\right. \\
& \left.+\left(\bar{\rho}_{i+\frac{1}{2}, j}^{r}-\bar{\rho}_{i+\frac{1}{2}, k}^{r}\right) \frac{h_{i+1, j}-h_{i, j}}{\Delta r}\right)-\frac{\nu_{\mathrm{h}}}{\Delta r}\left(h_{i+1, k} y_{1 ; i+1}\right. \\
& \left.\frac{u_{i+\frac{3}{2}, k}-u_{i+\frac{1}{2}, k}}{\Delta r}-h_{i, k} y_{1 ; i} \frac{u_{i+\frac{1}{2}, k}-u_{i-\frac{1}{2}, k}}{\Delta r}\right) \\
& +\alpha \bar{h}_{i+\frac{1}{2}, k}^{r} u_{i+\frac{1}{2}, k} \frac{v_{\mathrm{h}}}{r_{i+\frac{1}{2}}}-{\overline{y_{1 ; i+\frac{1}{2}}}}^{r}\left(v_{v ; i+\frac{1}{2}, k+\frac{1}{2}}\right. \\
& \left.\frac{u_{i+\frac{1}{2}, k+1}-u_{i+\frac{1}{2}, k}}{\bar{h}_{i+\frac{1}{2}, k+\frac{1}{2}}^{r z}}-v_{v ; i+\frac{1}{2}, k-\frac{1}{2}} \frac{u_{i+\frac{1}{2}, k}-u_{i+\frac{1}{2}, k-1}}{\bar{h}_{i+\frac{1}{2}, k-\frac{1}{2}}^{r z}}\right)=0
\end{aligned}
$$

Again, the alpha terms are marked with boxes. Another addition compared to the Cartesian 2-DV solution are the $y$ factors throughout the equation, which serve as width compensation factors. For $w$ momentum, a similar procedure is applied.

The governing equations are spatially discretized with a central differences approach, except for the advective terms. The advective terms are discretized with higher-order flux limiters (Fringer et al., 2005), namely MINMOD flux limiters in the case of the momentum equations and MUSCL flux limiters in the case of the transport equation.

The horizontal time integration of the momentum and transport equations is Euler explicit. The horizontal advective terms in the momentum equations are solved with the predictor-corrector scheme of MacCormack (Hirsch, 1988). The vertical time integration is semi-implicit, applying the $\theta$ scheme. The global continuity equation (Eq. 6) and 


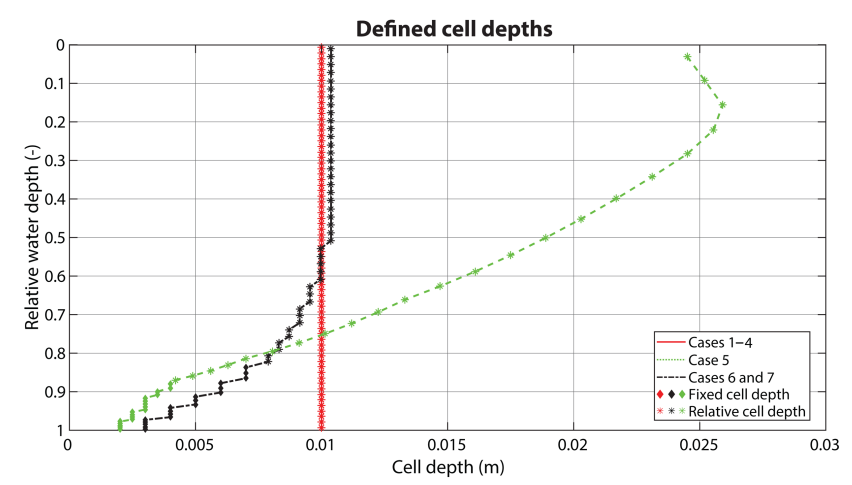

Figure 4. Defined cell depths for Cases 1 to 7. For plotting reasons, the vertical axis displays the depth from the water surface relative to the local water depth. The cell depths that are defined relative to the local water depth (as marked by ${ }^{*}$ ) are displayed for the average water depth in each case study.

barotropic forcing are solved semi-implicitly (Casulli and Cheng, 1992). The case studies (Sect. 2.4) apply an implicitness factor $\theta=1$ (i.e., the Euler implicit scheme) for the vertical momentum and transport equations, the global continuity equation, and the barotropic forcing. The non-hydrostatic pressure is standard solved with the Euler implicit scheme. The complete discretizations are shown in Appendix B.

The numerical framework largely follows the SWASH solution procedure (Zijlema et al., 2011). The code was extended by adding the alpha terms and factors accounting for the varying cell width in the tangential direction. The density and transport calculation modules were replaced by new modules based on the selected density equation (Wright, 1997) and the presented diffusivity equations.

\subsection{Verification and validation}

This article validates the model qualitatively and quantitatively. The behaviour of a local seepage inflow setting on double-diffusive layering is verified qualitatively (Sect. 2.4.3). The quantitative validation tests the model results against the following:

1. documented properties of systems of double-diffusive convection and salt fingering (Sect. 2.4.1);

2. and the expected expansion of an unconditionally stable layer near a bottom seepage inflow, for which an analytical solution is derived in Sect. 2.4.2.

In all the case studies (Table 1), we applied a time step of $2 \mathrm{~ms}$ and a horizontal mesh size of $5 \mathrm{~mm}$ in the radial direction. The vertical mesh size in Case 1 to Case 4 was set uniformly to $10 \mathrm{~mm}$. In Cases 5 to 7, the vertical mesh size varied over depth. Because the processes of most interest occurred near the bottom, the mesh size decreased towards the bottom (Fig. 4).

\subsubsection{Validation for double-diffusive characteristics}

This subsection lists several common metrics, which we applied to quantitatively validate our simulations of doublediffusive systems with varying density gradients (Cases 1 to 4). To validate the applicability of the standard $k-\epsilon$ model, we present model simulations for each of these cases both with and without the use of the turbulence model (Sects. 3.1 and 3.2).

The stability of a double-diffusive system is commonly expressed by its Turner angle $T u$ (Ruddick, 1983):

$T u=\arctan \left(\frac{N_{T}^{2}-N_{S}^{2}}{N_{T}^{2}+N_{S}^{2}}\right)$,

where the four-quadrant arctangent function preserves the sign of the density gradients, $N_{T}^{2}=-g \times \alpha_{\mathrm{V}} \times \partial T / \partial z$, and $N_{S}^{2}=g \times \beta_{\mathrm{V}} \times \partial S / \partial z \cdot \alpha_{\mathrm{V}}\left({ }^{\circ} \mathrm{C}^{-1}\right)$ and $\beta_{\mathrm{V}}\left(10^{3} \mathrm{~kg} \mathrm{~kg}^{-1}\right)$ are the volumetric expansion coefficients for temperature and salinity, respectively; the $z$ axis is in the downward direction. A stable system occurs for $|T u|<45^{\circ}$, whereas $|T u|>90^{\circ}$ yields a gravitationally unstable system. Double-diffusive convection occurs for $-90^{\circ}<T u<-45^{\circ}$, and salt fingering for $45^{\circ}<T u<90^{\circ}$.

The expansion coefficients $\alpha_{\mathrm{V}}$ and $\beta_{\mathrm{V}}$ vary with temperature and salinity and are calculated for the average salinity and temperature on the interface. We stress, however, that the calculation of density gradients is highly sensitive to the assumed values of $\alpha_{\mathrm{V}}$ and $\beta_{\mathrm{V}}$. The dependencies of the expansion coefficients on temperature and salinity $\left(T\left({ }^{\circ} \mathrm{C}\right)\right.$ and $S\left(10^{-3} \mathrm{~kg} \mathrm{~kg}^{-1}\right)$, respectively) are derived from a linear regression to the density derivatives to $T$ and $S$, and the density is calculated according to Wright (1997).

$$
\begin{aligned}
& \alpha_{\mathrm{V}}(T, S)=-2.289087 \times 10^{-5}+1.324960 \times 10^{-5} \\
& \quad \times T-9.289557 \times 10^{-8} \times T^{2}+1.563400 \times 10^{-6} \times S \\
& \beta_{\mathrm{V}}(T, S)=7.999302 \times 10^{-4}-2.777361 \times 10^{-6} \\
& \quad \times T+3.190719 \times 10^{-8} \times T^{2}-4.156012 \times 10^{-7} \times S
\end{aligned}
$$

Cases 1 and 2 concern a system with two layers of equal depth in which a cold and fresh water layer is overlying a warm and saline water layer (Table 1). Based on the Turner angle, double-diffusive convection is expected to occur. The onset of convection and salt and heat transport across the interface is induced by applying a few very small perturbations of order $10^{-6}{ }^{\circ} \mathrm{C}$ throughout the temperature field. Case 1 has a smaller density ratio $R_{\rho}=-N_{T}^{2} / N_{S}^{2}$ than Case 2 . Note that articles concerning double-diffusive convection commonly define $R_{\rho}$ as its reciprocal in contrast to the common density gradient calculations for salt-finger systems. For the sake of consistency, this article employs one definition of the density ratio (i.e., the thermal density gradient over the saline density gradient).

Based on the Turner angles of Cases 3 and 4 in which warm and saline water is overlying cold and fresh water, salt 
Table 1. The dimensions, properties, and consequent stability parameters applied in the case studies. Up and Down refer to the upper and lower layer of the dual-layered system (in Cases 5 to 7 , the lower temperatures and salinities are properties of the central inflow).

\begin{tabular}{|c|c|c|c|c|c|c|c|c|c|}
\hline \multirow{2}{*}{ Case } & \multicolumn{2}{|c|}{ Dimension (m) } & \multicolumn{2}{|c|}{$T\left({ }^{\circ} \mathrm{C}\right)$} & \multicolumn{2}{|c|}{$S$ (weight $-\%$ ) } & \multirow{2}{*}{$w_{\text {in }}\left(\mathrm{m} \mathrm{s}^{-1}\right)$} & \multirow{2}{*}{$T u\left(^{\circ}\right)$} & \multirow{2}{*}{$R_{\rho}-$} \\
\hline & Depth & Radial & Up & Down & $\mathrm{Up}$ & Down & & & \\
\hline 1 & 0.7 & 3.0 & 10 & 20 & 0 & 15 & - & -53.3 & 0.15 \\
\hline 2 & 0.7 & 3.0 & 10 & 34 & 0 & 15 & - & -71.6 & 0.50 \\
\hline 3 & 0.7 & 3.0 & 20 & 10 & 1 & 0 & - & 71.2 & 2.04 \\
\hline 4 & 0.7 & 3.0 & 20 & 15 & 1 & 0 & - & 85.0 & 1.19 \\
\hline 5 & 0.4 & 3.0 & 30 & 5 & 0 & 10 & $5 \times 10^{-4}$ & -13.2 & -0.62 \\
\hline 6 & 0.5 & 1.5 & 20 & 25 & 1 & 3 & $1 \times 10^{-3}$ & -82.5 & 0.77 \\
\hline 7 & 0.5 & 1.5 & 20 & 26 & 1 & 2.5 & $1 \times 10^{-3}$ & -96.4 & 1.25 \\
\hline
\end{tabular}

fingers are expected to occur (Table 1). Similar to the previous cases, we slightly perturbed the temperature field on a few locations. Case 3 has a larger density ratio than Case 4, yielding a lower salt flux over the interface (Kunze, 2003).

An effective transport of heat and salt over the interface while maintaining a sharp interface is expected as this is a known property of double-diffusive salt fingers (Turner, 1965). Care should be taken that these salt fingers are calculated in a 2-D radial grid. Yoshida and Nagashima (2003) pointed out that there is still a lack of knowledge about the 2-D and 3-D structures of salt fingers and its implications for the interpretation of 2-D numerical results.

A clear definition of the interface location is relevant for the determination of the boundary properties and the heat and salt flux across the boundary. In each simulation, the interface location $z_{\text {int }}$ is defined for each depth profile of $S$ and $T$ as the location of the isoscalar. The isoscalar is constant and defined as the average value of $S$ and $T$ across the initial interface. Figure 5 marks the interface locations for Cases 3 and 4 as the locations of the isoscalars for times $t=0$ and $t=6 \mathrm{~h}$.

The vertical saline and thermal density fluxes across the interface, $F_{c}$, are calculated on each grid location by time differentiating the salt and heat volumes above the interface according to Carpenter et al. (2012):

$F_{c}=\frac{\mathrm{d}}{\mathrm{d} t} \int_{0}^{z_{\mathrm{int}}} \rho_{c}(z) \mathrm{d} z$

where $\rho_{c}$ is the converted value of $S$ or $T$ to density units (i.e., $\rho_{0} \beta S$ or $\rho_{0} \alpha T$, with $\rho_{0}$ being the reference density for the average salinity and temperature at the interface).

The simulated salt and heat fluxes are compared with theoretical fluxes based on molecular diffusivities and doublediffusion-specific eddy diffusivities according to the equation (Carpenter et al., 2012)

$F_{c ; \text { theoretical }}=\left.D \frac{\mathrm{d} \rho_{c}}{\mathrm{~d} z}\right|_{z_{\text {int }}}$, with the derivative taken at each location of the isoscalar. Since this location is usually not located on the horizontal cell boundary, the derivative is determined by applying a weighted average to the derivatives at the neighbouring cell boundaries. $D$ can be either the molecular diffusivity $D_{\text {mol }}$ (Eqs. 10 and 11) or an eddy diffusivity. In this article, the eddy diffusivities are only calculated for salt diffusion by applying the following relationship with the molecular thermal diffusivity:

$D_{\text {eddy }} S=D_{\text {mol; } T} \frac{R_{\rho}}{\gamma}$,

with $\gamma=F_{T} / F_{S}$ being the ratio of the heat and salt fluxes. A large variety of theoretical equations have been proposed for the flux ratio $\gamma$, both for salt fingers (e.g., Stern, 1975),

$\gamma_{\text {Stern }}=R_{\rho}-\left(R_{\rho}\left(R_{\rho}-1\right)\right)^{\frac{1}{2}}$,

and for double-diffusive convection (e.g., Kelley , 1990; Fernando, 1989):

$$
\begin{aligned}
\gamma_{\text {Kelley }} & =\left(\frac{R_{\rho}^{-1}+1.4\left(R_{\rho}^{-1}-1\right)^{\frac{3}{2}}}{1+14\left(R_{\rho}^{-1}-1\right)^{\frac{3}{2}}}\right)^{-1}, \\
\gamma_{\text {Fernando }} & =\tau^{\frac{1}{2}} R_{\rho},
\end{aligned}
$$

where the Lewis number $\left(\tau=D_{\mathrm{mol} ; T} / D_{\mathrm{mol} ; S}\right)$ is the ratio of the molecular thermal and saline diffusivities.

For double-diffusive convection, we also compare the heat fluxes with theoretical heat fluxes as predicted by Kelley (1990) and Linden and Shirtcliffe (1978). 

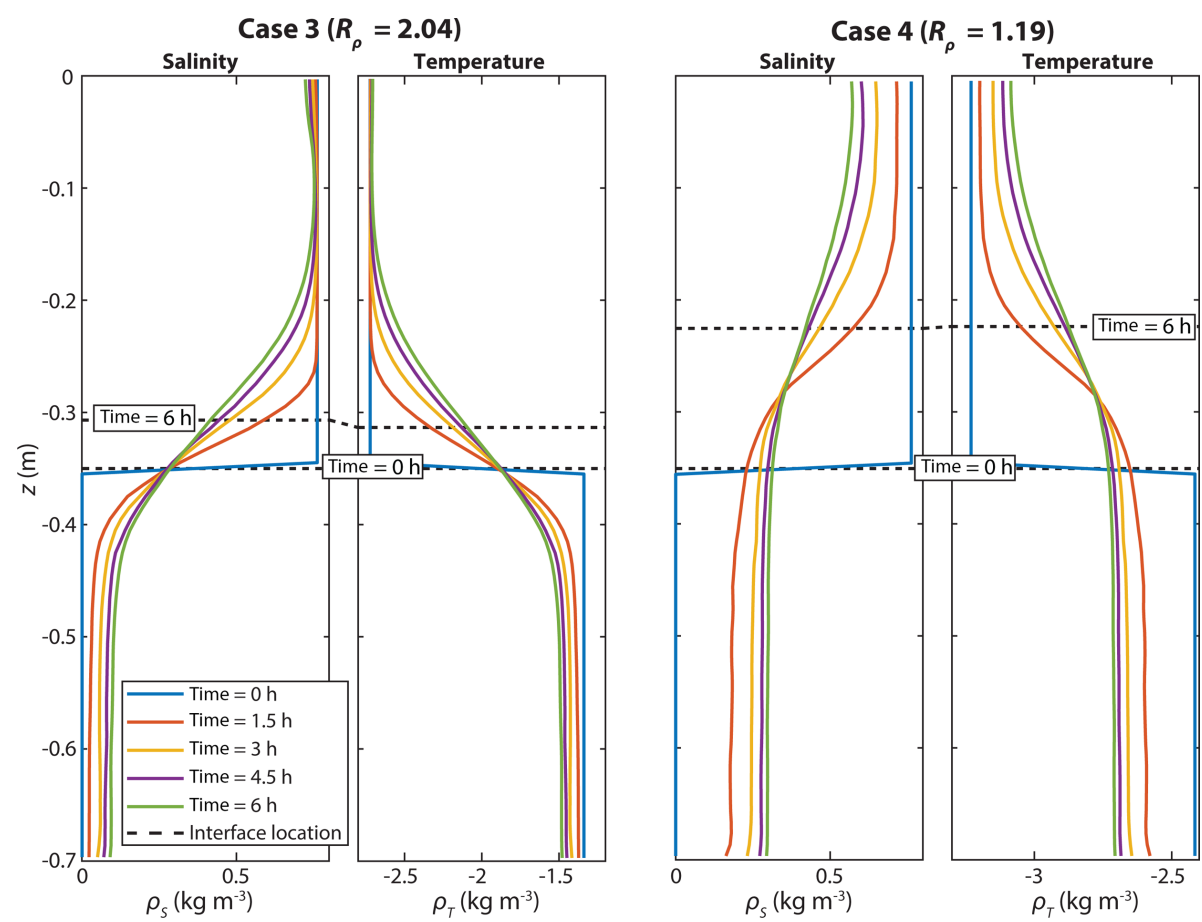

Figure 5. Interface positioning over time displayed on the depth profiles of $S$ and $T$ (both in $\mathrm{kg} \mathrm{m}^{-3}$ relative to the reference density) for the density ratios $R_{\rho}=2.04$ (Case 3) and $R_{\rho}=1.19$ (Case 4). The depth profiles are averaged over the complete horizontal domain and represent the simulations without a turbulence model.

$F_{T}^{\text {Kelley }}=0.0032 \exp \left(\frac{4.8}{R_{\rho}^{-0.72}}\right)\left(\frac{g D_{\text {mol } T}^{2}}{\rho_{0} v}\right)^{1 / 3} \rho_{T}^{4 / 3}$

$F_{T}^{\text {Linden \& Shirtcliffe }}=$

$\frac{1}{\left(\pi R a_{\mathrm{c}}\right)^{\frac{1}{3}}} \frac{\left(1-\tau^{-\frac{1}{2}} R_{\rho}^{-1}\right)^{\frac{4}{3}}}{\left(1-\tau^{-\frac{1}{2}}\right)^{\frac{1}{3}}}\left(\frac{g D_{\mathrm{mol} ; T}^{2}}{\rho_{0} v}\right)^{1 / 3} \rho_{T}^{4 / 3}$

The critical Rayleigh number is set to $R a_{\mathrm{c}}=10^{3}$. In line with the common practice in this field of study, the heat fluxes are presented as a ratio to the heat flux through a solid plane (Turner, 1973).

$F_{T}^{\mathrm{SP}}=0.085\left(\frac{g D_{\mathrm{mol} ; T}^{2}}{\rho_{0} \mathrm{v}}\right)^{1 / 3} \rho_{T}^{4 / 3}$

For systems of double-diffusive convection, we calculate the evolution of the boundary layer thicknesses $h_{c}$ according to Carpenter et al. (2012):

$h_{c}=\frac{\Delta \rho_{c}}{\left.\frac{\partial \rho_{c}}{\partial z}\right|_{z_{\mathrm{int}}},}$

where the density difference between the upper and lower layer, $\Delta \rho_{c}$, is determined for averaged values of $c$ over the upper and lower quarter of its depth profile. The ratio of boundary layer thicknesses $r$ scales to $\tau$ by the relation $r \sim \tau^{\frac{1}{5}}$ and is expected to approach 2.5 for salt-heat systems (Carpenter et al., 2012).

As a last validation metric for the salt-finger cases, we employ the Stern number (Stern, 1969):

$S t=\frac{F_{T}-F_{S}}{v\left(\frac{\partial \rho_{T}}{\partial z}-\frac{\partial \rho_{S}}{\partial z}\right)}$.

Stern suggested that the growth of salt fingers is arrested when $S t$ reaches $O(1)$. However, Stern numbers have been reported as varying from $O\left(10^{-3}\right)$ to $O\left(10^{2}\right)$ for finger systems. Recently, Traxler et al. (2011) reported Stern numbers $S t=9.4$ and $S t=76$ for DNS simulations with density ratios $R_{\rho}=2.0$ and $R_{\rho}=1.2$. These density ratios are comparable to Case 3 and Case 4, allowing us to compare our results with these DNS simulations.

\subsubsection{Analytical validation of a stable inflow}

The quantitative validation of an unconditionally stable bottom layer is based on an analytical solution for the radial expansion of this dense layer from a central inflow under laminar flow conditions (Case 5; Table 1). The interface expansion is described by its increasing interface radius $r_{\text {int }}$ over time. When the inflow is colder and more saline than the overlying water body, the developing layer has differ- 


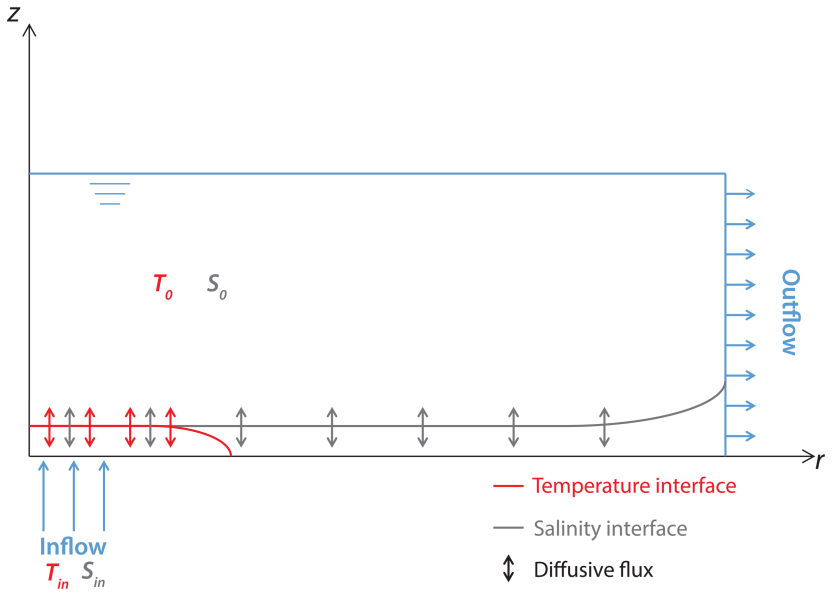

Figure 6. Conceptualization of the quantitative validation (Case 5), with locations of the salinity and temperature interfaces at a certain time after the start of a central inflow. The inflow is colder and more saline than the overlying water body.

ent growth rates for the salinity and temperature interface (Fig. 6). This is a consequence of the molecular heat diffusion, which is approximately 100 times larger than the diffusion of salt. In laminar flow conditions, molecular diffusion is the main driver of heat and salt exchange in stable layered systems.

In this quantitative case study, the central inflow has an outer radius of $0.2 \mathrm{~m}$. To allow for a slow development of the bottom layer, the inflow is placed slightly deeper compared to the rest of the bottom, and the inflow velocity linearly increases over the first $20 \mathrm{~min}$. The discharge over the right outflow boundary is set equal to the inflow discharge:

$Q_{\text {out }}=Q_{\text {in }}=w_{\text {in }} \times A_{\text {in }}$.

To derive the growth rates of the temperature and salinity interfaces, we consider the similarity solution of the heat equation for a fixed boundary concentration (Bergman et al., 2011):

$c(x, t)=c_{\text {in }}+\Delta c \times \operatorname{erfc}\left(\frac{x}{\sqrt{4 \times D \times t}}\right)$,

where $x$ is the distance from the interface and $\Delta c=c_{0}-c_{\text {in }}$ is the difference in concentrations (salinity or temperature) between the upper water body, represented by its initial concentration, and the inflow. The total mass $M$ that has crossed the interface is found through the integration of Eq. (33) over $x=0 \rightarrow \infty$ and multiplication of the growing interface surface $A_{\text {int }}$.

$$
\begin{aligned}
M(t) & =A_{\text {int }} \times \int_{0}^{\infty}\left(c-c_{\text {in }}\right) \mathrm{d} x \\
& =A_{\text {int }} \times \Delta c \times \int_{0}^{\infty} \operatorname{erfc}\left(\frac{x}{\sqrt{4 \times D \times t}}\right) \mathrm{d} x \\
& =A_{\text {int }} \times \Delta c \times \frac{\sqrt{4 \times D \times t}}{\pi}
\end{aligned}
$$

Derivation over time results in the time-dependent mass flux over the interface:

$\Phi_{\text {int }}(t)=\frac{\mathrm{d} M}{\mathrm{~d} t}=\Delta c \times \sqrt{\frac{D \times t}{\pi}} \times\left(2 \times \frac{\mathrm{d} A_{\text {int }}}{\mathrm{d} t}+\frac{A_{\text {int }}}{t}\right)$.

With $A_{\text {int }}=\pi r_{\text {int }}^{2}$ and assuming that the interface surface increases linearly with time at a constant inflow, we can rewrite the following:

$r_{\text {int }}(t)=\sqrt{\frac{\Phi_{\text {int }}}{3 \times \Delta c} \times \sqrt{\frac{t}{D \times \pi}}}$.

We assume that no mass is stored in the lower layer. Consequently, the mass flux that crosses the interface is equal to the net mass flux into the domain $\Phi_{\text {in }}-\Phi_{\text {out }} \approx w_{\text {in }} \times A_{\text {in }} \times$ $\left(c_{\text {in }}-c_{0}\right)$.

$r_{\text {int }}(t)=\sqrt{\frac{w_{\text {in }} \times A_{\text {in }}}{3} \times \sqrt{\frac{t}{D \times \pi}}}$

This equation can be used to validate the interface growth of both the salinity and temperature interface in the case of laminar flow.

\subsubsection{Verification for double-diffusive characteristics}

Cases 6 and 7 represent seepage inflows similar to the ones for which this modelling approach is developed. A duallayered system is built up by a central inflow through the bottom with an outer radius of $0.25 \mathrm{~m}$ (Table 1). The inflow velocity $w_{\text {in }}$ is built up linearly over the first $10 \mathrm{~min}$ to prevent a sudden pressure wave at $t=0$. Like Case 5 , the average water level is kept constant by a uniform outflow with the same discharge over the right outer boundary. Based on the Turner angle, a system with double-diffusive convection is expected to build up in Case 6, whereas a gravitationally unstable system is expected to develop in Case 7.

\section{Results and discussion}

The performance of the numerical framework was tested in several case studies subject to double-diffusive processes. The numerical results of these case studies and the extended SWASH code are presented in Hilgersom et al. (2017). 

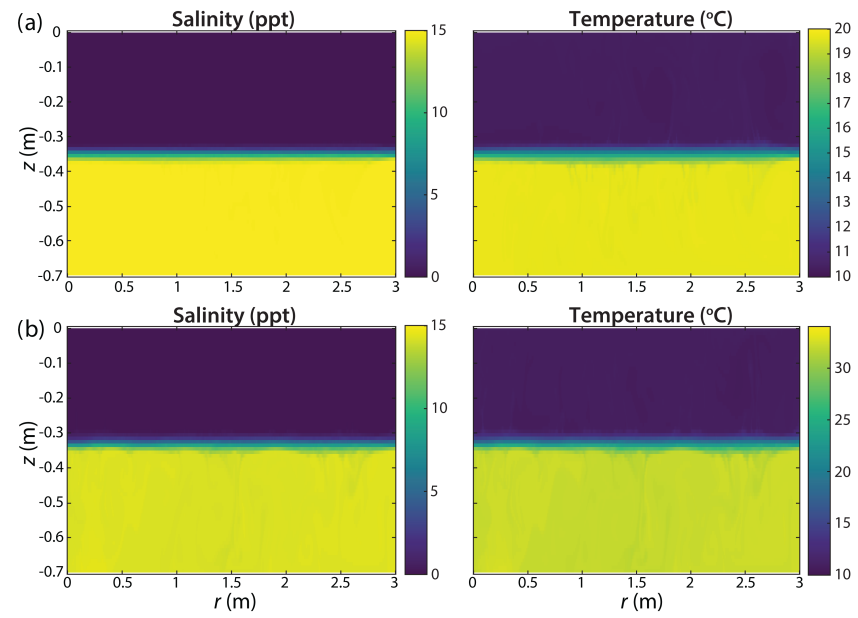

Figure 7. Double-diffusive convection in a layered system with a cold and fresh layer on top of warm and saline water $(t=4500 \mathrm{~s}$ since the start), with density ratios of (a) $R_{\rho}=0.15$ (Case 1) and (b) $R_{\rho}=0.50$ (Case 2). All figures represent simulations without the use of a turbulence model.

\subsection{Cases 1 and 2: double-diffusive convection}

The temperature and salinity gradients in Cases 1 and 2 yield a theoretical onset of double-diffusive convection, with respective Turner angles of -53.3 and $-71.6^{\circ}$. The numerical results confirm that a layered system is maintained that is bordered by a thin boundary layer from which unstable plumes emerge (Fig. 7). These are clear characteristics of double-diffusive convection.

The boundary layer thickness ratio $r$ is expected to be $\sim 2.5$. Figure 8 shows that none of the simulations for Cases 1 and 2 reach this value of $r$. For Case 1, the simulation without the aid of a turbulence model reaches the highest value of $r$, although this ratio starts to decrease again $3.5 \mathrm{~h}$ after the start. The fact that the expected values of $r$ are not reached during the simulations seems in line with Carpenter et al. (2012), who presented the evolution of $r$ in 3-D DNS simulations of a salt-heat system. They found that the salinity field in their simulation was not well resolved over the first $14 \mathrm{~h}$. Only after this first period of high turbulence does the boundary layer thickness ratio approach and remain at its expected value.

Regarding the tendency to reach a steady state step by step by building up a system with a stable boundary layer, our findings for Case 1 with a turbulence model seem more alarming. Here, $r$ falls back to a value of 1.18 after its first increase and remains at this value afterwards. The fact that the simulation does not tend to a system with a higher value of $r$ afterwards might indicate that the standard $k-\epsilon$ model does not function for systems with high density gradients.

The simulations for Case 2 also show an initial increase in $r$, followed by one or more drops. The density ratio $R_{\rho}=$ 0.50 indicates a mere turbulent system. In this sense, it is ex- pected that the boundary layers do not develop easily. Here, the simulation with a turbulence model seems to develop $r$ as expected over the first $1.5 \mathrm{~h}$. However, the ratio drops to values below $r=1.2$ afterwards.

The poor performance of the standard $k-\epsilon$ model also appears from the exaggerated salt and heat fluxes for Case 1 (Fig. 9b) compared to the simulation without the turbulence model (Fig. 9a). In the latter simulation, the heat transport over the interface appears to follow the theoretical heat transport as predicted from the molecular heat diffusion. Based on the flux ratio equations by Kelley (1990) and Fernando (1989) (Eqs. 25 and 26), we expect that the salt transport across the interface is lower than the heat transport. Initially, this is not the case (Fig. 9a), but the salt flux approaches the values predicted by Fernando (1989) after $110 \mathrm{~min}$. This moment coincides with the moment that $r$ starts a sharp increase in Fig. 8. In general, the salt flux has expected lower values than the heat flux over the period that the ratio $r$ is highest.

The ratio of the simulated heat fluxes to the $4 / 3$ flux law (Eqs. 27-29) shows a similar jump after 110 min (Fig. 10). From this moment, the simulated heat flux in Case 1 without a turbulence model temporarily approaches the predicted heat flux of Kelley (1990) and Linden and Shirtcliffe (1978). Again, a similar tendency is not visible for the simulation with a turbulence model, confirming that the standard $k-\epsilon$ model suppresses the onset of double-diffusive convection.

In line with the expectations for turbulent flows, the simulations for Case 2 show a large variation in heat and salt transport (Fig. 9). The simulations with and without a turbulence model both display a heat flux that is variably higher and lower than the salt flux, but displays the same pattern. Theoretically, the ratio of the turbulent heat and salt fluxes across the boundary approaches $\tau^{\frac{1}{2}}$ (Fernando, 1989) as $R_{\rho}$ approaches unity.

The dissimilar behaviour of our simulation with a turbulence model can be explained by the employed eddy diffusivities, which have similar values for salt and heat diffusion (note that the turbulent Prandtl and Schmidt number have similar values). These eddy diffusivities were not employed in the simulation without a turbulence model, which indicates that the similar heat and salt transport across the interface is caused by turbulent mixing through this interface. We refer to Sect. 3.6 for a further discussion on this in light of the employed standard $k-\epsilon$ model.

\subsection{Cases 3 and 4: salt fingers}

The numerical results for Case $3\left(T u=71.2^{\circ}\right)$ and Case 4 $\left(T u=85.0^{\circ}\right)$ confirm that salt fingers are formed over the interface (Fig. 11). The 3-D representation of these fingers would be circles around the centre of the axisymmetric grid, which is in disagreement with the real-world physics. However, the model does represent the upward- and downwardmoving parcels of water from the interface and consequently 

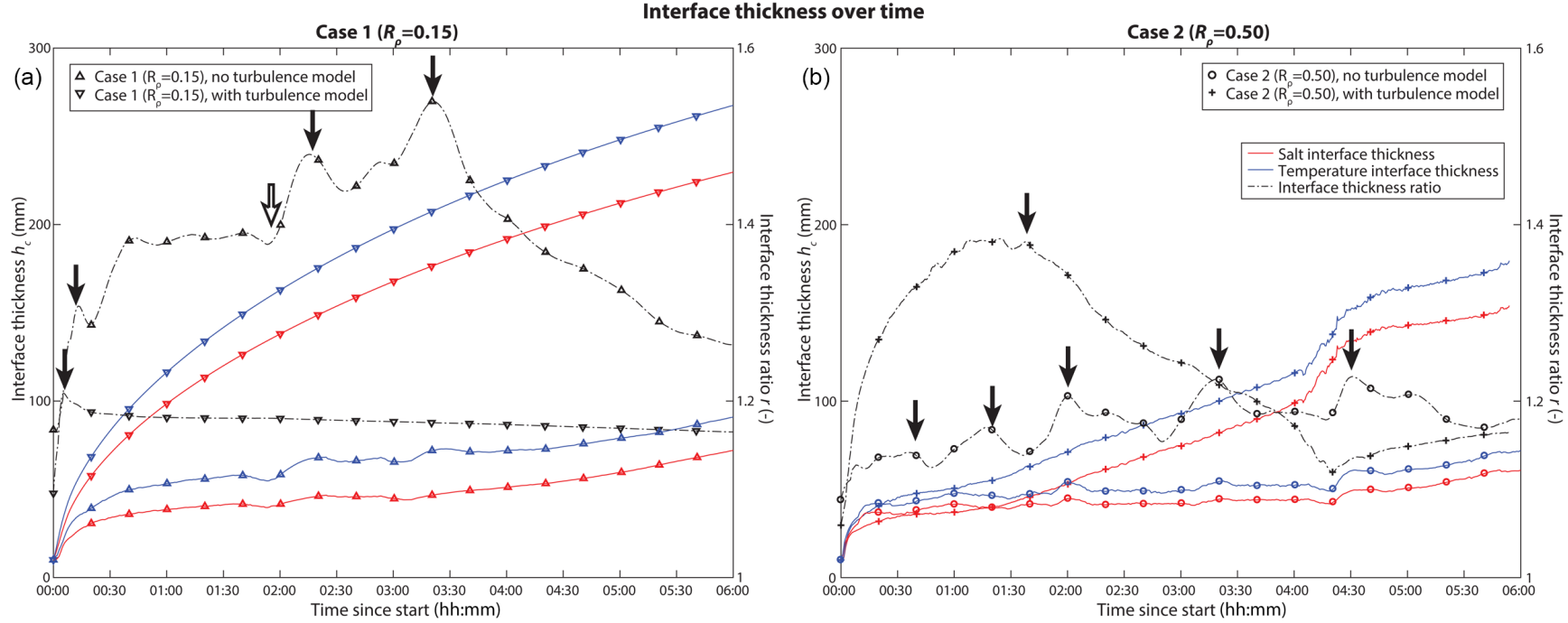

Figure 8. Time evolution of the interface thicknesses $r$ for the depth profiles of $S$ (red) and $T$ (blue) and their ratios for $R_{\rho}=0.15$ (Case 1 , a) and $R_{\rho}=0.50$ (Case 2, b). The interface thicknesses are averaged over the complete horizontal domain and each subplot presents the results for the simulations with and without a turbulence model. The filled arrows mark clear drops of $r$, and the open arrow marks the moment that $r$ starts its further increase in the direction of the theoretical interface ratio $r \approx 2.5$ in the simulation of Case 1 without a turbulence model.

results in reasonable measures of salt and heat transport across the interface.

Based on the difference in density ratios, the salt fingers in Case 4 are expected to transport more salt and heat than Case 3 (Sect. 2.4). Figure 5 shows an interface rise of about $0.04 \mathrm{~m}$ in Case 3 and $0.13 \mathrm{~m}$ in Case 4 over a numerical model run of $6 \mathrm{~h}$. Given the system of closed boundaries, we therefore find a significantly larger transport over the interface in Case 4.

Similar to Cases 1 and 2 (Fig. 9), we calculated the salt and heat fluxes across the interface for Case 3 and Case 4. For these cases, however, we only report the simulated flux ratios $\gamma_{\text {sim }}$ of the heat and salt fluxes (Fig. 12). For salt fingers, flux ratios lower than unity are expected from Eq. (24). From our simulations, however, we find flux ratios higher than unity. These flux ratios are more in line with oceanic values in which turbulent values of $\gamma$ can approach 1.6 (Kunze, 2003). Over the simulated $6 \mathrm{~h}$, the flux ratios show a decreasing tendency. However, particularly for the mere turbulent Case 3, we observe sudden upward jumps in the flux ratios preventing the flux ratios from reaching consistent values below unity over the course of the simulations. Based on these results, we hypothesize a settling of the system with more constant low flux ratios in the long run.

Based on a 3-D DNS model, Traxler et al. (2011) found Stern numbers $S t=9.4$ and $S t=76$ for $R_{\rho}=2.0$ and $R_{\rho}=$ 1.2 , respectively. Our simulations for $R_{\rho}=2.04$ (Case 3) and $R_{\rho}=1.19$ (Case 4) yield lower average Stern numbers of approximately 0.73 and 1.95 , respectively (Fig. 13). One reason for the lower values could be the fact that our model simulates salt and heat transport in two dimensions: Traxler et al. (2011) reported a Stern number of approximately 3.5 in 2-DV simulations for $R_{\rho}=2.0$. Although our simulations yield even lower Stern numbers, values around $S t=1$ are not uncommon for salt-finger systems.

\subsection{Case 5: radial expansion of a dense water layer}

The analytical solution for the radial expansion of inflowing cold and saline water (Eq. 39) holds for a situation with laminar flow. Given the geometric properties of the conceptualized situation and the initially very thin layer of dense water, it is difficult to define the inflow properties so that the flow near the inflow is immediately laminar. For the selected inflow parameters (Table 1), laminarization of the flow appears to occur after approximately 1700 s (Fig. 14). From that moment, the numerical results show significant differences between the salinity and temperature interface growth. The analytical results are therefore shifted in time to match the interface radii with the numerical results at the moment that the flow becomes laminar.

Accounting for a purely molecular diffusion, the numerical results show a fair agreement with the analytical results. As we found some small occasional eddies occurring after $t=1700 \mathrm{~s}$, we also plotted analytical results assuming the diffusivity was on average $0.2 \%$ influenced by turbulent diffusion. Here, the turbulent diffusion was calculated by dividing an assumed kinematic viscosity $v=10^{-6} \mathrm{~m}^{2} \mathrm{~s}^{-1}$ by the Prandtl-Schmidt number (Eqs. 8 and 9). The assumption of a slight influence of turbulence diffusion shows a better agreement with the numerical results.

One critical note here is the sensitivity of the interface growth to the definition of the interface location. Similar to the previous cases, we defined the interface location as 

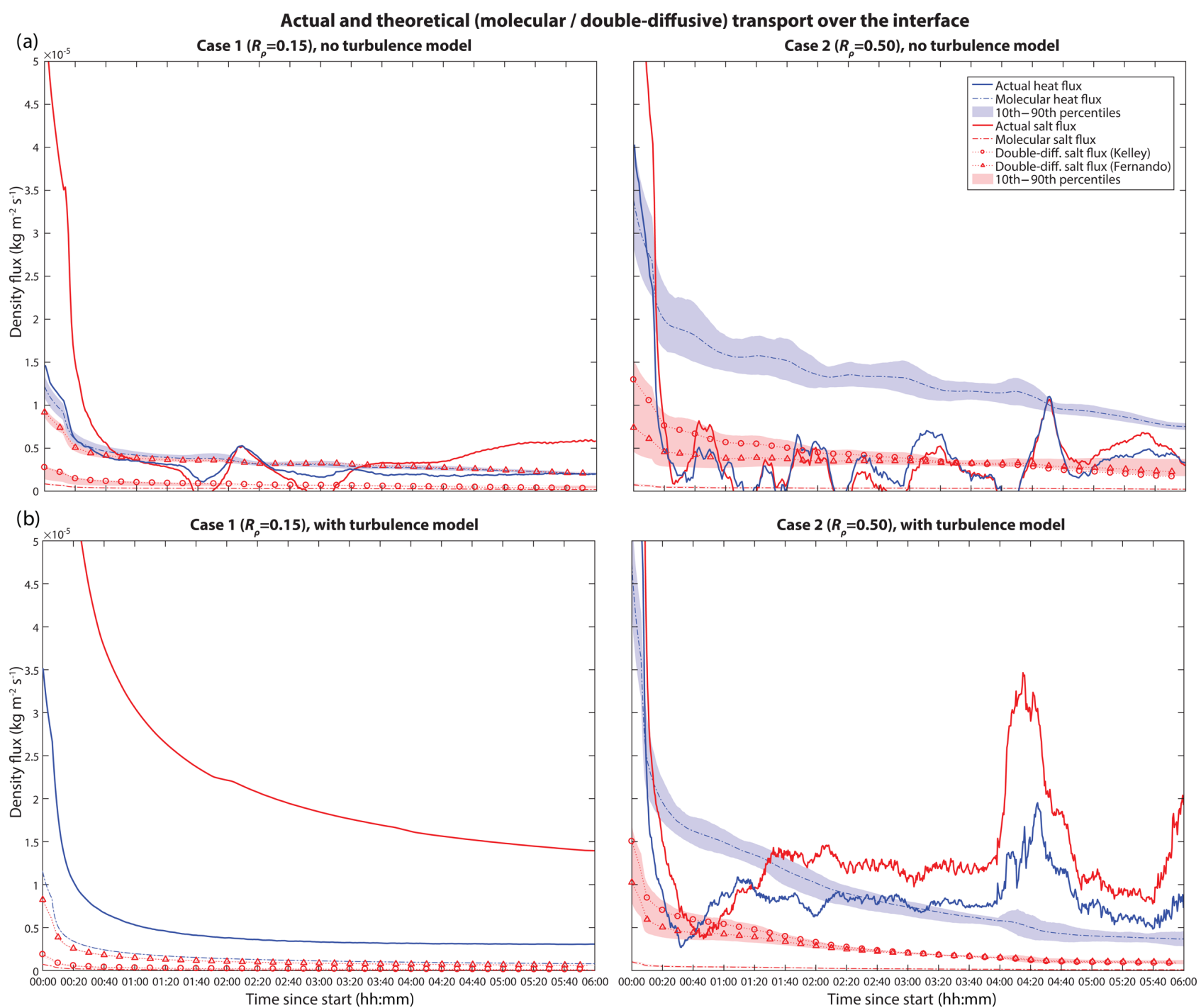

Figure 9. Time evolution of the salt (red) and temperature fluxes (blue) over the interface for $R_{\rho}=0.15$ (Case 1 , left) and $R_{\rho}=0.50$ (Case 2, right) and for (a) the simulations without a turbulence model and (b) the simulations with a turbulence model. The fluxes represent horizontal averages throughout the complete domain. The figures also present theoretical fluxes over the interface, which were calculated from molecular and double-diffusive diffusivities, and their uncertainty bounds for horizontal variations in density gradients at the interface.

halfway to the step change between the inflow concentration $\left(T_{\text {in }}\right.$ and $\left.S_{\text {in }}\right)$ and the concentration of the water body $\left(T_{0}\right.$ and $S_{0}$ ) because this matches our visual interpretation of the interface in the numerical results. However, selecting the interface at a larger percentage of the step change significantly increases the growth and makes the numerical and analytical results incomparable.

\subsection{Case 6: inflow yielding double-diffusive convection}

The temperature and salinity gradients in Case 6 yield the onset of double-diffusive convection. Like Cases 1 to 4, a sharp interface develops over which salt and heat is transported by diffusion. Figure 15 confirms the development of a salt-heat interface and a convective layer above the boil. Other con- vective cells further transport the salt and heat above the interface. Figure 15 shows that a considerable amount of heat and salt was already conveyed to the upper layer over the first $1.5 \mathrm{~h}$. The lower convective layer slowly builds up, and local eddies clearly counteract the development when the lower convective layer is still thin.

\subsection{Case 7: gravitationally unstable inflow}

Compared to Case 6, a slightly altered inflow temperature and salinity in Case 7 theoretically makes the developing layer gravitationally unstable (Table 1). In other words, the water body itself is denser than the inflowing water, which consequently flows upwards. The numerical results confirm the onset of a central buoyant flow above the inflow (Fig. 16). 
Flux law comparison (ratios against heat transport through a solid plane)

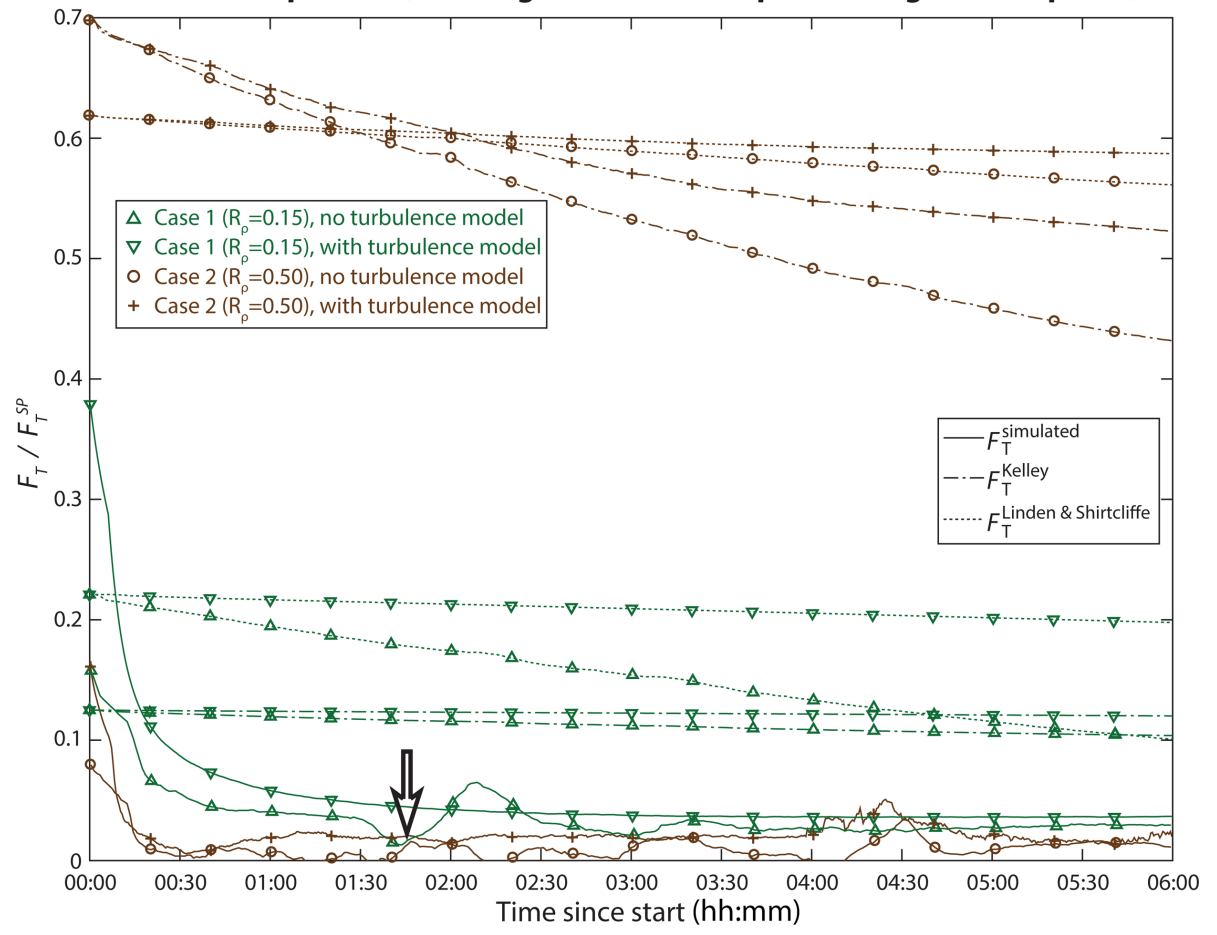

Figure 10. Simulated and theoretical heat fluxes according to Eqs. (27) and (28) relative to the theoretical heat flux through a solid plane (Eq. 29). Green colours represent the results for Case 1 and brown colours represent the results for Case 2. The open arrow marks the moment that the simulated heat flux starts rising towards the theoretical flux laws in the simulation of Case 1 without a turbulence model.
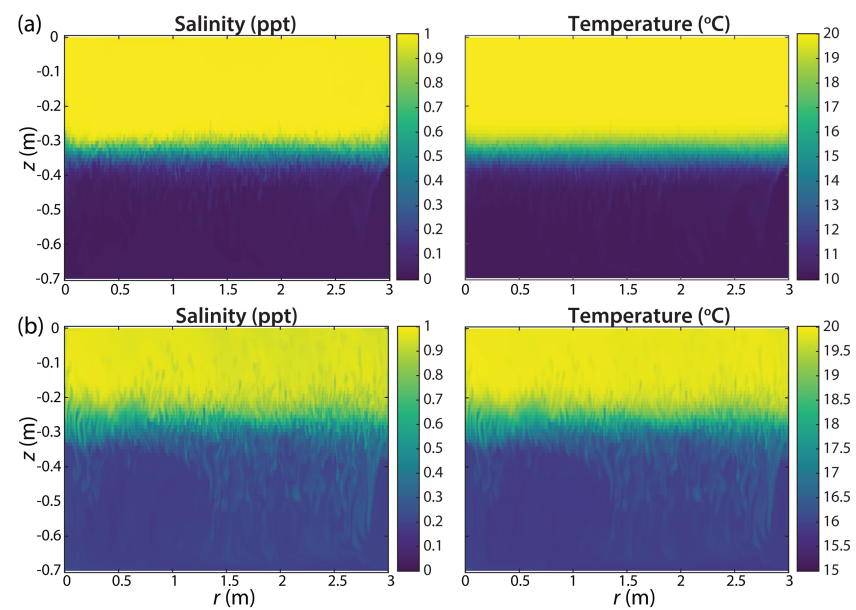

Figure 11. Salt fingering in a layered system with warm and saline water on top of a cold and fresh layer $(t=4500 \mathrm{~s}$ since the start), with density ratios of (a) $R_{\rho}=2.04$ (Case 3) and (b) $R_{\rho}=1.19$ (Case 4). All figures represent simulations without the use of a turbulence model.

Interestingly, plumes develop from the upward flow. Downward plumes are also visible below the floating warm and saline water. Like the salt fingers in Cases 3 and 4, in which warm and saline water also overlaid cold and fresh water, this is a mechanism to dissipate the heat and salt gradients.

\subsection{Turbulence model}

In the previous subsections, we found that the standard $k-$ $\epsilon$ model performed insufficiently accurately for predicting the production and dissipation of turbulence. This subsection briefly discusses the performances of the turbulence model and future prospects for a relatively simple improvement of the model.

In Sect. 3.1, we found a similar heat and salt transport across the interface that was likely caused by turbulent mixing at the interface. Following this hypothesis, the RANS model apparently requires a turbulence model that suppresses the turbulence across the interface, but predicts the onset of turbulence in the unstable regions near the interface. This is a known defect of the standard $k-\epsilon$ model, which does not account for buoyancy effects near strong density gradients. Section 3.3 also stressed the importance of the right timing with which turbulence is modelled when flows are variably laminar and turbulent.

More advanced turbulence models have been developed for systems with large density gradients (e.g., Venayagamoorthy, 2003; Paik et al., 2009). Toffolon et al. (2015) recently showed how a minimal model with two parameters is 


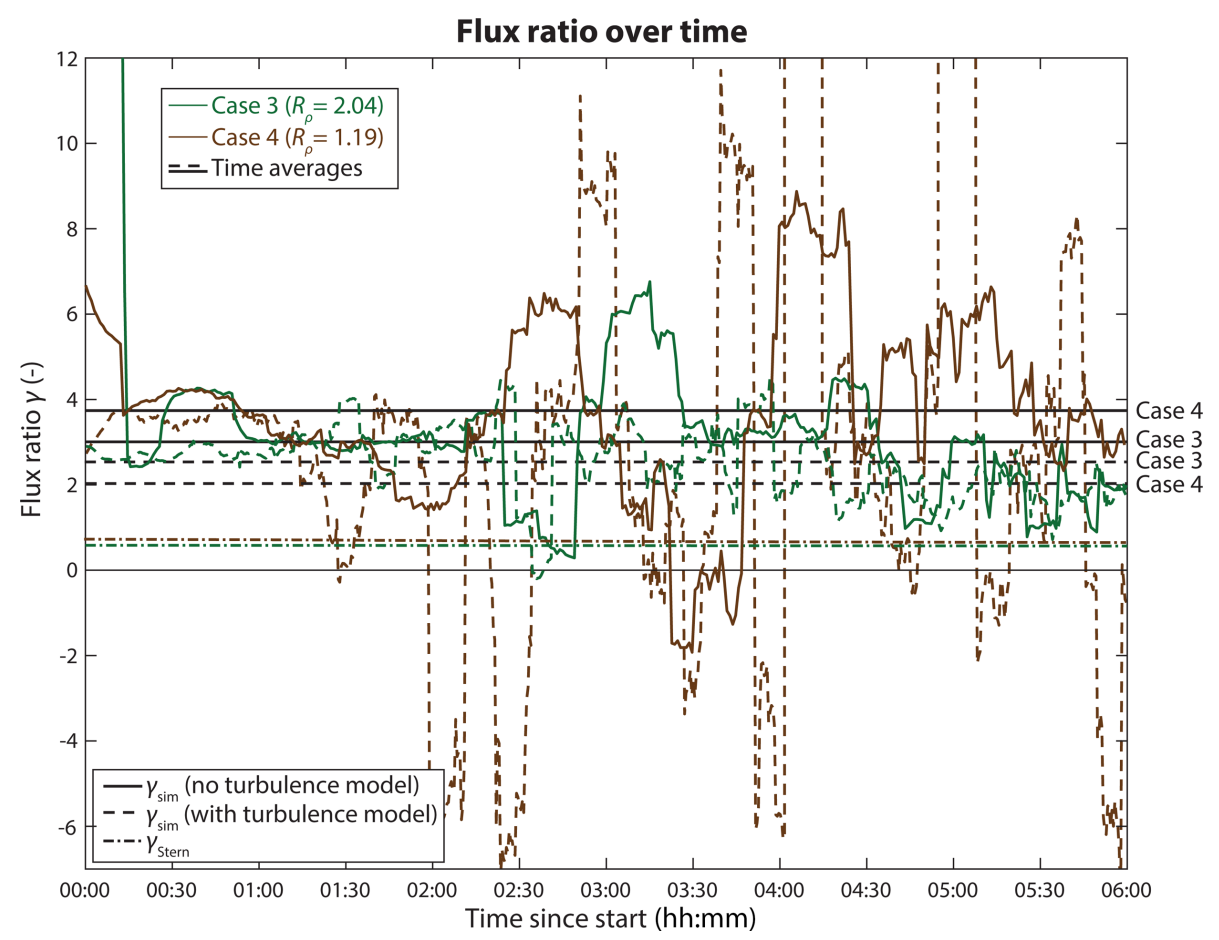

Figure 12. The evolution of flux ratios over time for $R_{\rho}=2.04$ (Case 3, green) and $R_{\rho}=1.19$ (Case 4 , brown). Continuous lines mark the simulations without a turbulence model, and dotted lines marks the simulations with a turbulence model. The black lines mark the temporal averages for each simulation.

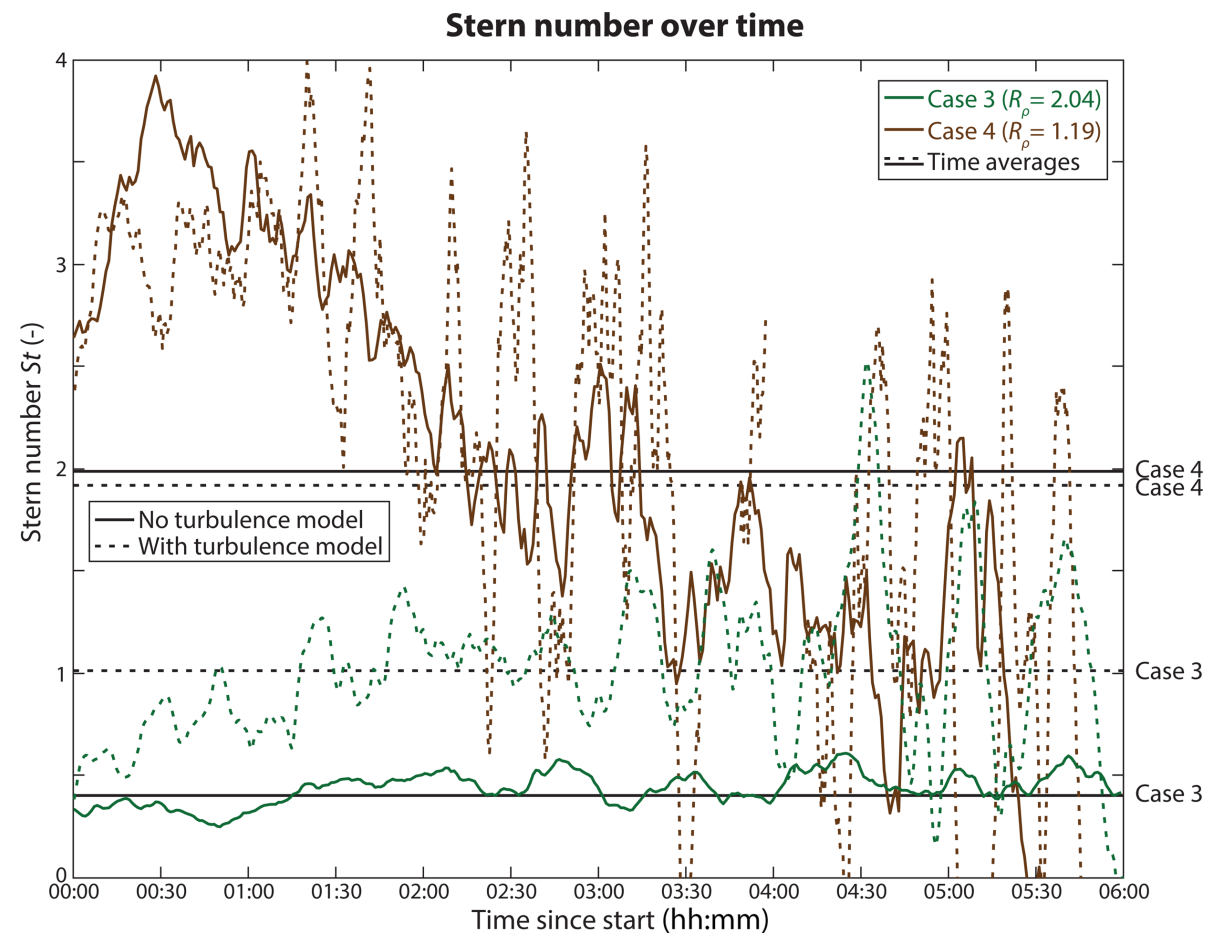

Figure 13. The evolution of the Stern numbers over time for $R_{\rho}=2.04$ (Case 3, green) and $R_{\rho}=1.19$ (Case 4, brown). Continuous lines mark the simulations without a turbulence model, and dotted lines marks the simulations with a turbulence model. The black lines mark the temporal averages for each simulation. 

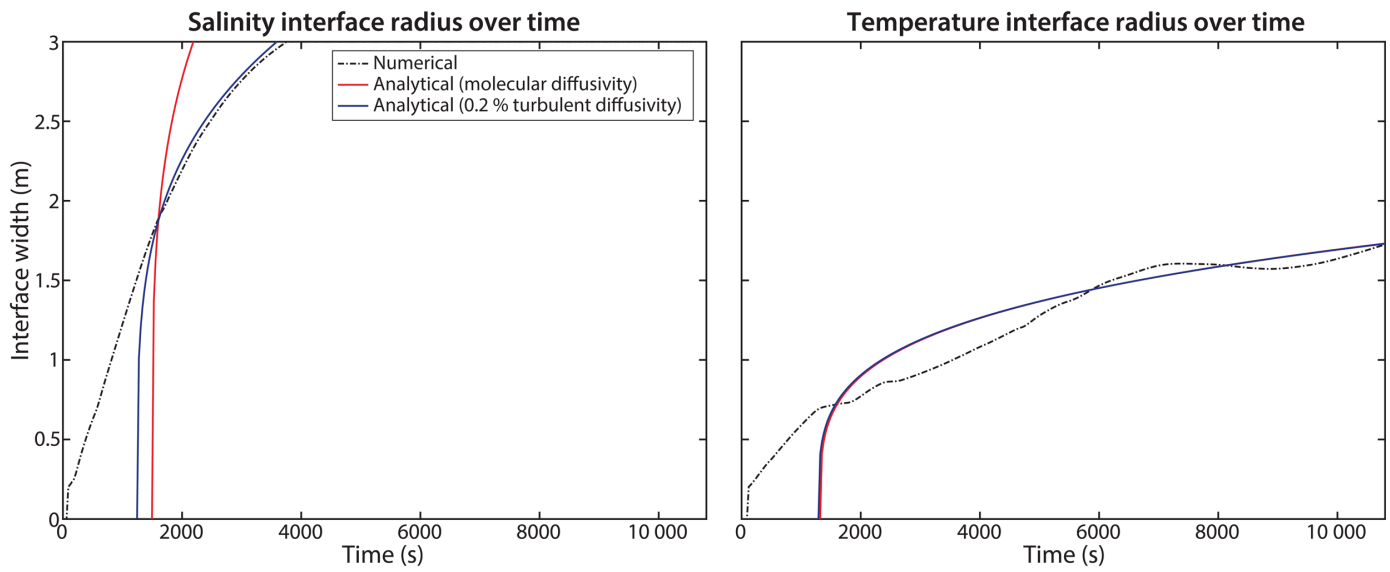

Figure 14. Evolution of the interface between a warm and fresh water body and a bottom cold and saline layer developing form a central inflow (Case 5). After $t=1700 \mathrm{~s}$, the flow in the numerical results becomes laminar and differences between the temperature and salinity interface growth become visible. Analytical results are plotted for the assumptions of completely molecular diffusion (red) and for diffusivities that are $0.2 \%$ influenced by turbulent diffusion (blue).
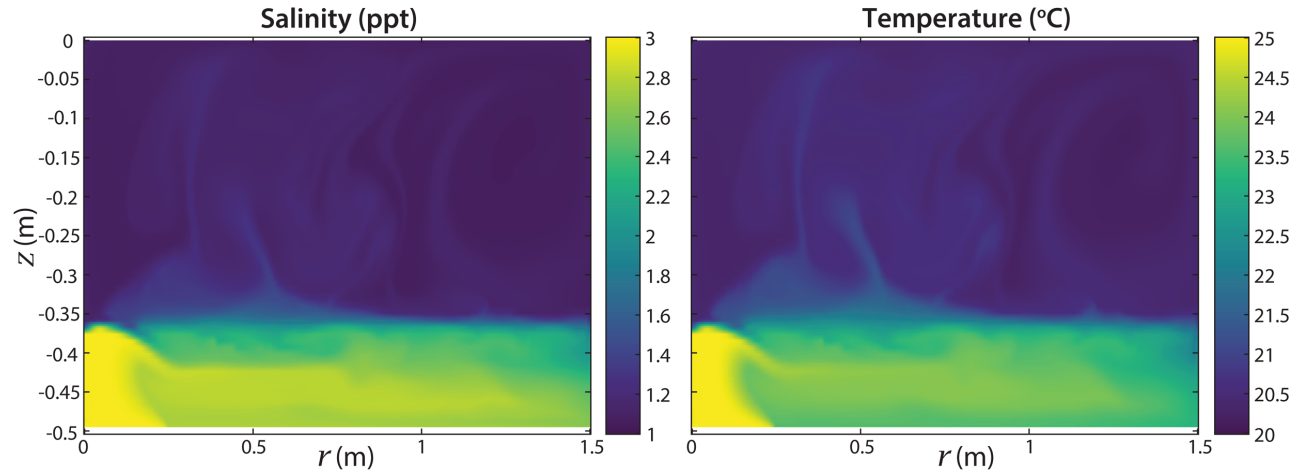

Figure 15. Double-diffusive layering (Case 6) with cold and fresh water on top of a warm and saline inflow ( $t=5400 \mathrm{~s}$ since the start).

already able to characterize differences in transport between sharp interfaces and unstable regions in a thermohaline staircase. Extending the $k-\epsilon$ model of SWASH with a parameterization that accounts for these distinct regions could yield a large improvement in properly representing turbulence on proper locations and times. With such an advanced turbulence model, the largest improvements are expected when $R_{\rho}$ approaches unity, as a good turbulence model becomes increasingly important for these density gradients.

\section{Conclusions}

This article reports the successful derivation of an axisymmetric framework for a hydrodynamic model incorporating salt and heat transport. This model set-up allows us to efficiently calculate salt and heat transport whenever a situation is modelled that can be approximated by axisymmetry around a central location. The 2-D axisymmetric grid description demands approximately the same execution time as a regular 2-DV description with the same dense mesh and therefore avoids the need to solve the equations over a dense mesh in the third spatial dimension.

For our purpose of studying shallow water bodies, three aspects were important: (1) the inclusion of a free surface, (2) the efficient solution of a circular seepage inflow, which makes the problem three-dimensional, and (3) a proper simulation of density-driven flow and double-diffusivity-driven salt and heat transport. The former aspect was already fulfilled by employing the SWASH framework.

The second aspect was solved by assuming axisymmetry for the Reynolds-averaged Navier-Stokes equation in cylindrical coordinates. The derived numerical framework is presented as a Cartesian 2-DV description with a few additional terms and width compensation factors. Our implementation of these terms in the non-hydrostatic SWASH model demonstrates the opportunity to easily extend a 2-DV model towards the presented 2-D axisymmetric model.

The third aspect was fulfilled by extending SWASH with a new density and diffusivity module. The case studies 

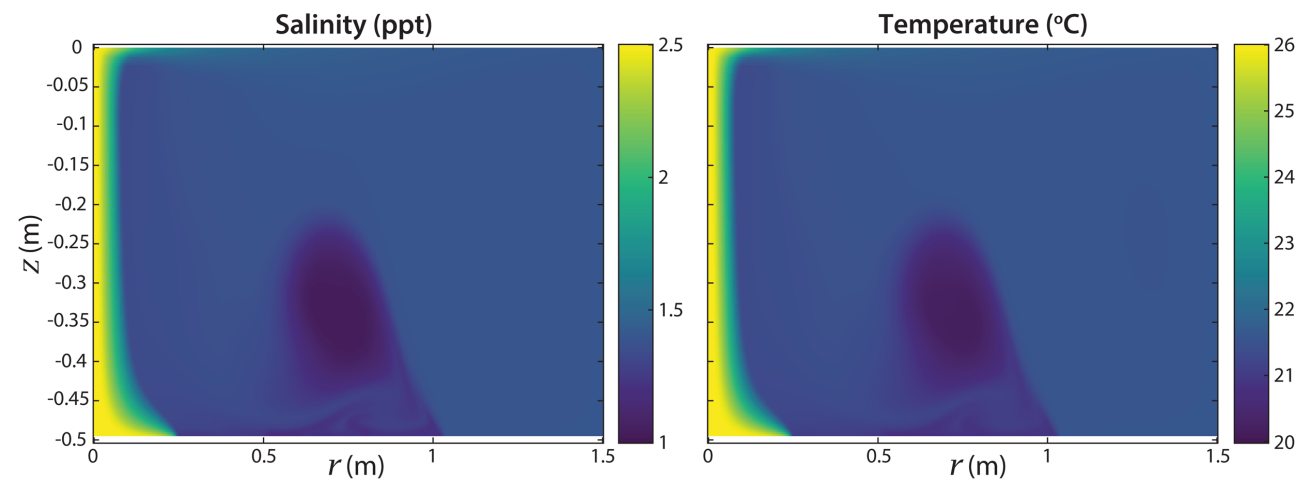

Figure 16. Unstable system (Case 7) with denser cold and fresh water on top of a warm and saline inflow $(t=5400 \mathrm{~s}$ since the start). The inflowing water flows upward through the centre independent of the inflow velocity.

demonstrate explainable behaviour for density-driven flow and double-diffusivity-driven salt and heat transport. The formation of convective layers and salt fingers is in accordance with the theory of double diffusivity and the enhanced salt and heat fluxes across the interface for density gradients approaching unity. Other validation metrics show that the RANS model does not meet the expected flux ratios and stability criteria in all cases, which is hypothesized to be caused by a defective turbulence modelling for systems of large density gradients. Replacing the standard $k-\epsilon$ model with an advanced turbulence model might improve the results for these merely turbulent cases.

An analytic validation method was presented to evaluate the model's performance for a cold and saline inflow developing a dense water layer near the bottom. For laminar flow conditions, the numerical model showed a similar radial expansion of the bottom layer as expected from analytical results.
Although the model is already able to show expected behaviour in the double-diffusive regime, we recommend a further exploration of its limitations and possibilities. For example, a grid convergence study should indicate whether the selected mesh size yields a convergence of results for all diffusion- and advection-dominated cases. Further, a nearer comparison with DNS model results would support the validation of the model. In future applications, we stress that this model approach should be employed as a RANS model that simulates thermohaline stratification processes on a larger scale. As such, the model can be favourable in applications that allow for an axisymmetric approach.

Code and data availability. The model data for the five case studies and the extended SWASH code are accessible at https://doi.org/10.4121/uuid:95227d5d-2cf0-44ec-ab2d705a626dcdf4 (Hilgersom et al., 2017). 


\section{Appendix A: Cell depth integration with the Leibniz integral rule}

When the continuity, momentum, and transport equations are integrated over the cell depth, the Leibniz integral rule is applied to the time derivatives and the horizontal spatial derivatives. Here, we show the cell depth integration of $\frac{\partial u}{\partial t}$ and $\frac{\partial u u}{\partial r}$.

$\int_{z_{k-\frac{1}{2}}}^{z_{k+\frac{1}{2}}} \frac{\partial u}{\partial t} \mathrm{~d} z=\frac{\partial u_{k} h_{k}}{\partial t}-\left.u \frac{\partial z}{\partial t}\right|_{z_{k-\frac{1}{2}}} ^{z_{k+\frac{1}{2}}}$
$\int_{z_{k-\frac{1}{2}}}^{z_{k+\frac{1}{2}}} \frac{\partial u u}{\partial r} \mathrm{~d} z=\frac{\partial u_{k} u_{k} h_{k}}{\partial r}-\left.u \hat{u} \frac{\partial z}{\partial r}\right|_{z_{k-\frac{1}{2}}} ^{z_{k+\frac{1}{2}}}$

The derivatives $\frac{\partial u r}{\partial r}, \frac{\partial w}{\partial t}, \frac{\partial u w}{\partial r}, \frac{\partial p}{\partial r}, \frac{\partial c}{\partial t}$, and $\frac{\partial c}{\partial r}$ in Eqs. (1), (2), (3), and (7) are integrated in a similar fashion.

\section{Appendix B: Full discretizations}

\section{B1 U momentum}

$$
\begin{aligned}
& \frac{u_{i+\frac{1}{2}, k}^{n+\theta_{u}}}{\theta_{u} \Delta t}+\frac{{\overline{\omega_{i+\frac{1}{2}, k+\frac{1}{2}}^{n}}}^{r}\left(\widehat{u}_{i+\frac{1}{2}, k+\frac{1}{2}}^{n+\theta_{u}}-u_{i+\frac{1}{2}, k}^{n+\theta_{u}}\right)}{\overline{h_{i+\frac{1}{2}, k}^{n} r}}-\frac{{\overline{\omega_{i+\frac{1}{2}, k-\frac{1}{2}}^{n}}}^{r}\left(\widehat{u}_{i+\frac{1}{2}, k-\frac{1}{2}}^{n+\theta_{u}}-u_{i+\frac{1}{2}, k}^{n+\theta_{u}}\right)}{\overline{h_{i+\frac{1}{2}, k}^{n}} r}-v_{v ; i+\frac{1}{2}, k+\frac{1}{2}}^{n} \frac{u_{i+\frac{1}{2}, k+1}^{n+\theta_{u}}-u_{i+\frac{1}{2}, k}^{n+\theta_{u}}}{\bar{h}_{i+\frac{1}{2}, k}^{n} \overline{h_{i+\frac{1}{2}, k+\frac{1}{2}}^{n}} r z}
\end{aligned}
$$

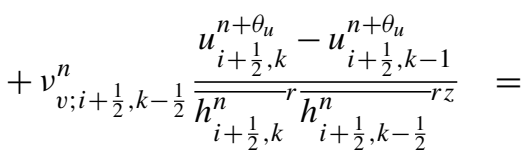

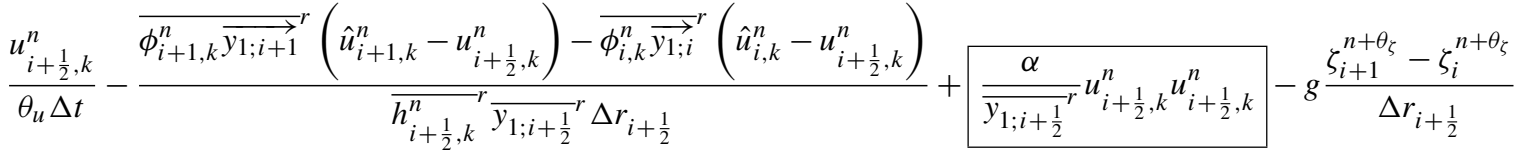

$$
\begin{aligned}
& -\frac{q_{i+1, k}^{n} h_{i+1, k}^{n} y_{1 ; i+1}-q_{i, k}^{n} h_{i, k}^{n} y_{1 ; i}}{\overline{h_{i+\frac{1}{2}, k}^{n}} r \overline{y_{1 ; i+\frac{1}{2}}} \Delta r_{i+\frac{1}{2}}}+\overline{q_{i+\frac{1}{2}, k+\frac{1}{2}}^{n}} \frac{z_{i+1, k+\frac{1}{2}}^{n}-z_{i, k+\frac{1}{2}}^{n}}{{\overline{h_{i+\frac{1}{2}, k}^{n}}}^{n} \Delta r_{i+\frac{1}{2}}}-\overline{q_{i+\frac{1}{2}, k-\frac{1}{2}}^{n}} r \frac{z_{i+1, k-\frac{1}{2}}^{n}-z_{i, k-\frac{1}{2}}^{n}}{{\overline{h_{i+\frac{1}{2}, k}^{n}}}^{n} \Delta r_{i+\frac{1}{2}}}+\frac{\alpha \overline{\bar{q}_{i+\frac{1}{2}, k}^{n}}}{{\overline{y_{1 ; i+\frac{1}{2}}}}^{r}}
\end{aligned}
$$

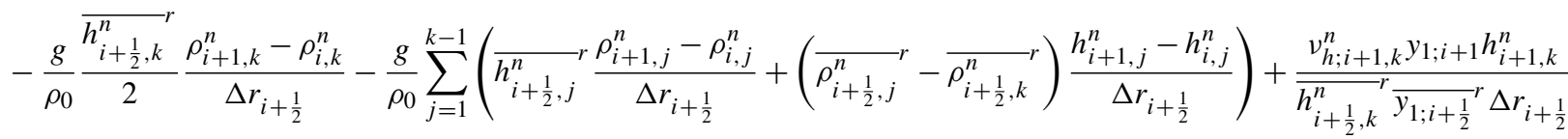

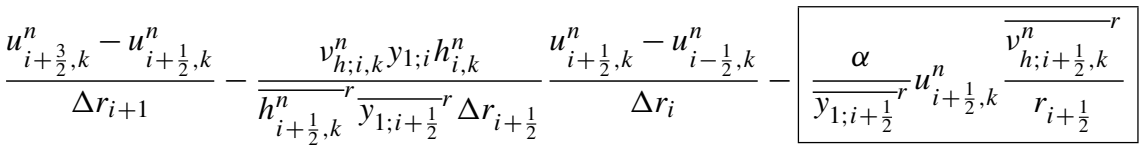




\section{B2 $W$ momentum}

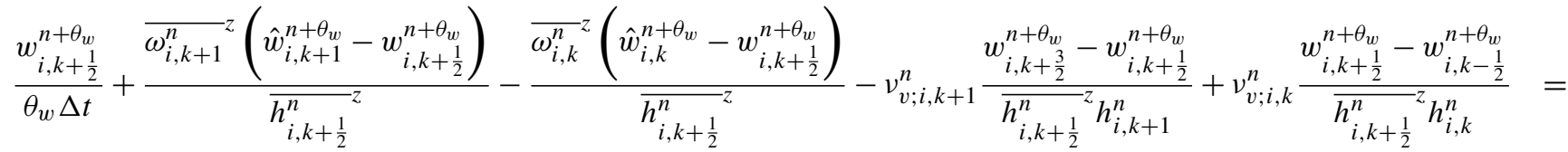

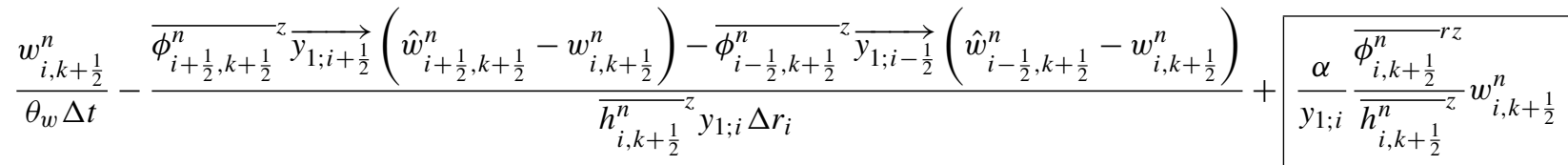

$$
\begin{aligned}
& -\frac{q_{i, k+1}^{n}}{{\overline{h_{i, k+\frac{1}{2}}^{n}}}^{n}}+\frac{q_{i, k}^{n}}{{\overline{h_{i, k+\frac{1}{2}}^{n}}}^{n}}+\frac{v_{h ; i+\frac{1}{2}, k+\frac{1}{2}}^{n} \bar{y}_{1 ; i+\frac{1}{2}} r{\overline{h_{i-\frac{1}{2}, k+\frac{1}{2}}^{n}}}^{r z}}{{\overline{h_{i, k+\frac{1}{2}}^{n}}}^{n} y_{1 ; i} \Delta r_{i}} \frac{w_{i+1, k+\frac{1}{2}}^{n}-w_{i, k+\frac{1}{2}}^{n}}{\Delta r_{i+\frac{1}{2}}}-\frac{v_{h ; i-\frac{1}{2}, k+\frac{1}{2}}^{n} \bar{y}_{1 ; i-\frac{1}{2}}^{r} \overline{h_{i-\frac{1}{2}, k+\frac{1}{2}}^{n}}}{{\overline{h_{i, k+\frac{1}{2}}^{n}}}^{z} y_{1 ; i} \Delta r_{i}} \\
& \frac{w_{i, k+\frac{1}{2}}^{n}-w_{i-1, k+\frac{1}{2}}^{n}}{\Delta r_{i-\frac{1}{2}}}
\end{aligned}
$$

\section{B3 Transport equation}

$$
\begin{aligned}
& \frac{c_{i, k}^{n+1}}{\Delta t}+\frac{\omega_{i, k+\frac{1}{2}}^{n+1} \hat{c}_{i, k+\frac{1}{2}}^{n+1}}{h_{i, k}^{n+1}}-\frac{\omega_{i, k-\frac{1}{2}}^{n+1} \hat{c}_{i, k-\frac{1}{2}}^{n+1}}{h_{i, k}^{n+1}}-\frac{D_{v ; i, k+\frac{1}{2}}}{h_{i, k}^{n+1}} \frac{c_{i, k+1}^{n+1}-c_{i, k}^{n+1}}{\overline{h_{i, k+\frac{1}{2}}^{n+1}} z}+\frac{D_{v ; i, k-\frac{1}{2}}}{h_{i, k}^{n+1}} \frac{c_{i, k}^{n+1}-c_{i, k-1}^{n+1}}{\overline{h_{i, k-\frac{1}{2}}^{n+1} z}}=
\end{aligned}
$$

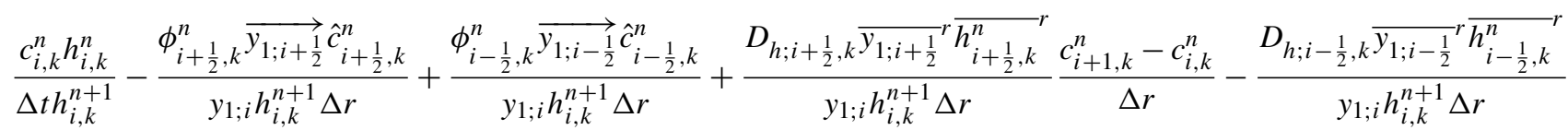

$$
\begin{aligned}
& \frac{c_{i, k}^{n}-c_{i-1, k}^{n}}{\Delta r}-\frac{D_{h ; i+\frac{1}{2}, k} \overline{y_{1 ; i+\frac{1}{2}}} r \overline{h_{i+\frac{1}{2}, k}^{n}} r}{y_{1 ; i} h_{i, k}^{n+1} \Delta r} \frac{\overline{c_{i+\frac{1}{2}, k+1}^{n}}-\overline{c_{i+\frac{1}{2}, k-1}^{n}} r}{{\overline{h_{i+\frac{1}{2}, k+\frac{1}{2}}^{n}}}^{n}+\overline{h_{i+\frac{1}{2}, k-\frac{1}{2}}^{n}}} \frac{\overline{z_{i+1, k}^{n}}-\overline{z_{i, k}^{n}} z}{\Delta r}+\frac{D_{h ; i-\frac{1}{2}, k} \overline{y_{1 ; i-\frac{1}{2}}} r \overline{h_{i-\frac{1}{2}, k}^{n}}}{y_{1 ; i} h_{i, k}^{n+1} \Delta r}
\end{aligned}
$$

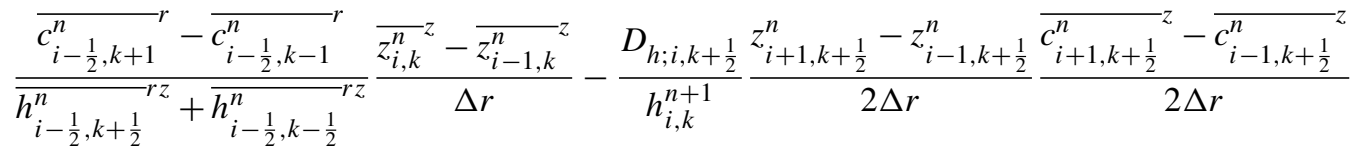

$$
\begin{aligned}
& +\frac{D_{h ; i, k+\frac{1}{2}}}{h_{i, k}^{n+1}}\left(\frac{z_{i+1, k+\frac{1}{2}}^{n}-z_{i-1, k+\frac{1}{2}}^{n}}{2 \Delta r}\right)^{2} \frac{c_{i, k+1}^{n}-c_{i, k}^{n}}{\bar{h}_{i, k+\frac{1}{2}}^{n}}-\frac{D_{h ; i, k-\frac{1}{2}}}{h_{i, k}^{n+1}}\left(\frac{z_{i+1, k-\frac{1}{2}}^{n}-z_{i-1, k-\frac{1}{2}}^{n}}{2 \Delta r}\right)^{2} \frac{c_{i, k}^{n}-c_{i, k-1}^{n}}{\bar{h}_{i, k-\frac{1}{2}}^{n}}
\end{aligned}
$$


Competing interests. The authors declare that they have no conflict of interest.

Acknowledgements. This project has been funded by the Netherlands Organisation for Scientific Research (NWO), project number 842.00.004.

Edited by: Ignacio Pisso

Reviewed by: three anonymous referees

\section{References}

Arnon, A., Lensky, N. G., and Selker, J. S.: High-resolution temperature sensing in the Dead Sea using fiber optics, Water Resour. Res., 50, 1756-1772, https://doi.org/10.1002/2013WR014935, 2014.

Batchelor, G.: An introduction to fluid dynamics, Cambridge University Press, 615 pp., 1967.

Bennett, G., Reilly, T., and Hill, M.: Technical training notes in ground-water hydrology: radial flow to a well, Technical report, US Geological Survey; Books and Open-File Reports, 1990.

Bergman, T. L., Incropera, F. P., and Lavine, A. S.: Fundamentals of heat and mass transfer, John Wiley \& Sons, 1048 pp., 2011.

Berthold, S. and Börner, F.: Detection of free vertical convection and double-diffusion in groundwater monitoring wells with geophysical borehole measurements, Environ. Geol., 54, 15471566, https://doi.org/10.1007/s00254-007-0936-y, 2008.

Carpenter, J., Sommer, T., and Wüest, A.: Simulations of a doublediffusive interface in the diffusive convection regime, J. Fluid Mech., 711, 411-436, https://doi.org/10.1017/jfm.2012.399, 2012.

Casulli, V. and Cheng, R. T.: Semi-implicit finite difference methods for three-dimensional shallow water flow, Int. J. Numer. Meth. Fl., 15, 629-648, https://doi.org/10.1002/fld.1650150602, 1992.

Casulli, V. and Stelling, G.: Numerical simulation of 3D quasihydrostatic, free-surface flows, J. Hydraul. Eng.-ASCE, 124, 678-686, 1998.

Cathcart, T. P. and Wheaton, F. W.: Modeling temperature distribution in freshwater ponds, Aquacult. Eng., 6, 237-257, https://doi.org/10.1016/0144-8609(87)90021-5, 1987.

De Louw, P., Vandenbohede, A., Werner, A., and Oude Essink, G.: Natural saltwater upconing by preferential groundwater discharge through boils, J. Hydrol., 490, 74-87, https://doi.org/10.1016/j.jhydrol.2013.03.025, 2013.

Dias, J. and Lopes, J.: Implementation and assessment of hydrodynamic, salt and heat transport models: The case of Ria de Aveiro Lagoon (Portugal), Environ. Modell. Softw., 21, 1-15, https://doi.org/10.1016/j.envsoft.2004.09.002, 2006.

Eckart, C. H.: The equation of state of water and sea water at low temperatures and pressures, Part 2 of Properties of water, Am. J. Sci., 256, 225-240, 1958.

Fernando, H. J. S.: Buoyancy transfer across a diffusive interface, J. Fluid Mech., 209, 1-34, 1989.

Fringer, O., Armfield, S., and Street, R.: Reducing numerical diffusion in interfacial gravity wave simulations, Int. J. Numer. Meth. Fl., 49, 301-329, 2005.
Galletti, C., Parente, A., and Tognotti, L.: Numerical and experimental investigation of a mild combustion burner, Combust. Flame, 151, 649-664, 2007.

Giestas, M., Pina, H. L., Milhazes, J. P., and Tavares, C.: Solar pond modeling with density and viscosity dependent on temperature and salinity, Int. J. Heat Mass Tran., 52, 2849-2857, https://doi.org/10.1016/j.ijheatmasstransfer.2009.01.003, 2009.

Hilgersom, K., Van de Giesen, N., De Louw, P., and Zijlema, M.: Three-dimensional dense distributed temperature sensing for measuring layered thermohaline systems, Water Resour. Res. 52, 6656-6670, https://doi.org/10.1002/2016WR019119, 2016.

Hilgersom, K., Zijlema, M., and van de Giesen, N.: An axisymmetric hydrodynamical model: model code and data (v2), TU Delft, https://doi.org/10.4121/uuid:95227d5d-2cf044ec-ab2d-705a626dcdf4, 2017.

Hirsch, C.: Numerical Computation of Internal and External Flows, Wiley, Chichester, 1988.

Huppert, H. E. and Turner, J.: Double-diffusive convection, J. Fluid Mech., 106, 299-329, 1981.

Kelley, D. E.: Fluxes through diffusive staircases: a new formulation, J. Geophys. Res., 95, 3365-3371, https://doi.org/10.1029/JC095iC03p03365, 1990.

Kelley, D. E., Fernando, H. J. S., Gargett, A. E., Tanny, J., and Özsoy, E.: The diffusive regime of double-diffusive convection, Prog. Oceanogr., 56, 461-481, https://doi.org/10.1016/S00796611(03)00026-0, 2003.

Kimura, S. and Smyth, W.: Direct numerical simulation of salt sheets and turbulence in a double-diffusive shear layer, Geophys. Res. Lett., 34, L21610, https://doi.org/10.1029/2007GL031935, 2007.

Kimura, S., Smyth, W., and Kunze, E.: Turbulence in a sheared, saltfingering-favorable environment: Anisotropy and effective diffusivities, J. Phys. Oceanogr., 41, 1144-1159, 2011.

Kunze, E.: A review of oceanic salt-fingering theory, Prog. Oceanogr., 56, 399-417, https://doi.org/10.1016/S00796611(03)00027-2, 2003.

Langevin, C. D.: Modeling Axisymmetric Flow and Transport, Ground Water, 46, 579-590, https://doi.org/10.1111/j.17456584.2008.00445.x, 2008.

Launder, B. and Spalding, D.: The numerical computation of turbulent flows, Comput. Method Appl. M., 3, 269-289, https://doi.org/10.1016/0045-7825(74)90029-2, 1974.

Linden, P. F. and Shirtcliffe, T. G. L.: The diffusive interface in double-diffusive convection, J. Fluid Mech., 87, 417-432, https://doi.org/10.1017/S002211207800169X, 1978.

Menguc, M. and Viskanta, R.: Radiative transfer in axisymmetric, finite cylindrical enclosures, J. Heat Transf., 108, 271-276, 1986.

Noguchi, T. and Niino, H.: Multi-layered diffusive convection, Part 1: Spontaneous layer formation, J. Fluid Mech., 651, 443-464, https://doi.org/10.1017/S0022112009994150, 2010a.

Noguchi, T. and Niino, H.: Multi-layered diffusive convection, Part 2: Dynamics of layer evolution, J. Fluid Mech., 651, 465-481, https://doi.org/10.1017/S0022112010994160, 2010b.

Paik, J., Eghbalzadeh, A., and Sotiropoulos, F.: Three-dimensional unsteady RANS modeling of discontinuous gravity currents in rectangular domains, J. Hydraul. Eng., 135, 505-521, 2009.

Radko, T., Bulters, A., Flanagan, J., and Campin, J.-M.: Double-diffusive recipes, Part I: Large-scale dynamics of 
thermohaline staircases, J. Phys. Oceanogr., 44, 1269-1284, https://doi.org/10.1175/JPO-D-13-0155.1, 2014a.

Radko, T., Flanagan, J., Stellmach, S., and Timmermans, M.-L.: Double-diffusive recipes, Part II: Layer-merging events, J. Phys. Oceanogr., 44, 1285-1305, https://doi.org/10.1175/JPO-D-130156.1, 2014b.

Reilly, T. E. and Harbaugh, A. W.: Simulation of Cylindrical Flow to a Well Using the US Geological Survey Modular FiniteDifference Ground-Water Flow Model, Ground Water, 31, 489494, 1993.

Ruddick, B.: A practical indicator of the stability of the water column to double-diffusive activity, Deep-Sea Res. Pt. I, 30, 11051107, 1983.

Ruddick, B. and Gargett, A. E.: Oceanic doubleinfusion: introduction, Prog. Oceanogr., 56, 381-393, https://doi.org/10.1016/S0079-6611(03)00024-7, 2003.

Schmid, M., Lorke, A., Wüest, A., Halbwachs, M., and Tanyileke, G.: Development and sensitivity analysis of a model for assessing stratification and safety of Lake Nyos during artificial degassing, Ocean Dynam., 53, 288-301, 2003.

Schmid, M., Lorke, A., Dinkel, C., Tanyileke, G., and Wüest, A.: Double-diffusive convection in Lake Nyos, Cameroon, Deep-Sea Res. Pt. I, 51, 1097-1111, https://doi.org/10.1016/j.dsr.2004.02.010, 2004.

Schubert, F., Peiffer, A., Köhler, B., and Sanderson, T.: The elastodynamic finite integration technique for waves in cylindrical geometries, J. Acoust. Soc. Am., 104, 2604-2614, 1998.

Shima, E., Matsuda, T., Takeda, H., and Sawada, K.: Hydrodynamic calculations of axisymmetric accretion flow, Mon. Not. R. Astron. Soc., 217, 367-386, https://doi.org/10.1093/mnras/217.2.367, 1985.

Sommer, T., Carpenter, J. R., and Wüest, A.: Double-diffusive interfaces in Lake Kivu reproduced by direct numerical simulations, Geophys. Res. Lett., 41, 5114-5121, https://doi.org/10.1002/2014GL060716, 2014.

Stern, M. E.: Lateral mixing of water masses, Deep-Sea Res., 14, 747-753, https://doi.org/10.1016/S0011-7471(67)80011-1, 1967.

Stern, M. E.: Collective instability of salt fingers, J. Fluid Mech., 35, 209-218, https://doi.org/10.1017/S0022112069001066, 1969.

Stern, M. E.: Ocean circulation physics, Academic Press Inc., 1975.

Stommel, H. and Fedorov, K.: Small scale structure in temperature and salinity near Timor and Mindanao, Tellus A, 19, 306-325, https://doi.org/10.1111/j.2153-3490.1967.tb01484.x, 1967.

Stommel, H., Arons, A., and Blanchard, D.: An oceanographical curiosity: the perpetual salt fountain, Deep-Sea Res., 3, 152-153, 1956.

Suárez, F., Tyler, S., and Childress, A.: A fully coupled, transient double-diffusive convective model for salt-gradient solar ponds, Int. J. Heat Mass Tran., 53, 1718-1730, https://doi.org/10.1016/j.ijheatmasstransfer.2010.01.017, 2010.
Suárez, F., Ruskowitz, J. A., Childress, A. E., and Tyler, S. W.: Understanding the expected performance of large-scale solar ponds from laboratory-scale observations and numerical modeling, Appl. Energ., 117, 1-10, https://doi.org/10.1016/j.apenergy.2013.12.005, 2014.

SWASH: SWASH source code (Version 1.20), available at: http:// swash.sourceforge.net/ (last access: 30 May 2016), 2011.

Toffolon, M., Wüest, A., and Sommer, T.: Minimal model for double diffusion and its application to Kivu, Nyos, and Powell Lake, J. Geophys. Res.-Oceans, 120, 6202-6224, https://doi.org/10.1002/2015JC010970, 2015.

Traxler, A., Stellmach, S., Garaud, P., Radko, T., and Brummeln, N.: Dynamics of fingering convection. Part 1 Small-scale fluxes and large-scale instabilities, J. Fluid Mech., 677, 530-553, https://doi.org/10.1017/jfm.2011.98, 2011.

Turner, J. S.: The coupled turbulent transports of salt and heat across a sharp density interface, Int. J. Heat Mass Tran., 8, 759-760, 761-767, https://doi.org/10.1016/0017-9310(65)90022-0, 1965.

Turner, J. S.: Buoyancy Effects in Fluids, Cambridge University Press, 367 pp., 1973.

UNESCO: Tenth report of the joint panel on oceanographic tables and standards, UNESCO Technical Paper in Marine Science, vol. 36, 25 pp., 1981.

Vega, L. A.: Ocean Thermal Energy Conversion Primer, Mar. Technol. Soc. J., 36, 25-35, https://doi.org/10.4031/002533202787908626, 2002.

Venayagamoorthy, S. K., Koseff, J. R., Ferziger, J. H., and Shih, L. H.: Testing of RANS turbulence models for stratified flows based on DNS data, Stanford University CA Environmental Fluid Mechanics Lab, 2003.

Washburn, E. W. and West, C. J.: International Critical Tables of Numerical Data, Physics, Chemistry and Technology, vol. 5, McGraw-Hill, 471 pp., 1933.

Wright, D. G.: An Equation of State for Use in Ocean Models: Eckart's Formula Revisited, J. Atmos. Ocean. Tech., 14, 735-740, https://doi.org/10.1175/15200426(1997)014<0735:AEOSFU>2.0.CO;2, 1997.

Yoshida, J. and Nagashima, H.: Numerical experiments on salt-finger convection, Prog. Oceanogr., 56, 435-459, https://doi.org/10.1016/S0079-6611(03)00032-6, 2003.

Zijlema, M. and Stelling, G.: Efficient computation of surf zone waves using the nonlinear shallow water equations with nonhydrostatic pressure, Coast. Eng., 55, 780-790, 2008.

Zijlema, M. and Stelling, G. S.: Further experiences with computing non-hydrostatic free-surface flows involving water waves, Int. J. Numer. Meth. Fl., 48, 169-197, https://doi.org/10.1002/fld.821, 2005.

Zijlema, M., Stelling, G., and Smit, P.: SWASH: An operational public domain code for simulating wave fields and rapidly varied flows in coastal waters, Coast. Eng., 58, 992-1012, https://doi.org/10.1016/j.coastaleng.2011.05.015, 2011. 